\title{
SUMMATION-BY-PARTS OPERATORS FOR NON-SIMPLY CONNECTED DOMAINS*
}

\author{
SAMIRA NIKKAR ${ }^{\dagger}$ AND JAN NORDSTRÖM ${ }^{\dagger}$
}

\begin{abstract}
We construct fully discrete stable and accurate numerical schemes for solving partial differential equations posed on non-simply connected spatial domains. The schemes are constructed using summation-by-parts operators in combination with a weak imposition of initial and boundary conditions using the simultaneous approximation term technique. In the theoretical part, we consider the two-dimensional constant coefficient advection equation posed on a rectangular spatial domain with a hole. We construct the new scheme and study well-posedness and stability. Once the theoretical development is done, the technique is extended to more complex non-simply connected geometries. Numerical experiments corroborate the theoretical results and show the applicability of the new approach and its advantages over the standard multiblock technique. Finally, an application using the linearized Euler equations for sound propagation is presented.
\end{abstract}

Key words. initial boundary value problems, stability, well-posedness, boundary conditions, non-simply connected domains, complex geometries

AMS subject classification. 65M99

DOI. $10.1137 / 18 \mathrm{M} 1163671$

1. Introduction. High order summation-by-parts (SBP) operators, together with a weak imposition of initial and well-posed boundary conditions using the simultaneous approximation term (SAT) technique, provide provably fully discrete unconditionally stable schemes for steady or time-dependent spatial domains $[1,2,3,5]$. These schemes have so far been mostly developed for spatial domains consisting of simply connected regions. To handle more complicated geometries, hybrid formulations utilizing finite volume and finite difference methods $[6,7,8,9]$ have been proposed. Other alternatives within the finite difference community for complex geometries include finite difference schemes using over-set mesh discretizations [10,11, 12, 13], multiblock techniques $[14,15,16,17]$, as well as SBP extensions to unstructured grids $[18,19,20]$.

In this article, we extend the SBP-SAT technique to handle partial differential equations posed on non-simply connected multidimensional geometries. As a prototype problem, we consider rectangles with rectangular holes inside, where the holes are not part of the computational domain. Hybrid methods, discontinuous Galerkin methods, and other types of schemes of SBP-SAT form are easily applicable to nonsimply connected domains. In these methods, one divides the spatial domain into patches and glues the numerical solutions together via interface couplings. In this paper, we proceed the other way around and discretize the geometry patch surrounding the hole directly, without subdividing. The novelty of the new technique is that it reduces the number of block-to-block connections and in that way improves the accuracy and lowers the cost.

The rest of the article proceeds as follows. In section 2, we study the twodimensional constant coefficient advection equation posed on a rectangular geometry

\footnotetext{
${ }^{*}$ Submitted to the journal's Methods and Algorithms for Scientific Computing section January 3, 2018; accepted for publication (in revised form) January 23, 2018; published electronically May 1, 2018.

http://www.siam.org/journals/sisc/40-3/M116367.html

${ }^{\dagger}$ Department of Mathematics, Computational Mathematics, Linköping University, SE-581 83 Linköping, Sweden (samira.nikkar@gmail.com, jan.nordstrom@liu.se).
}

A1250 

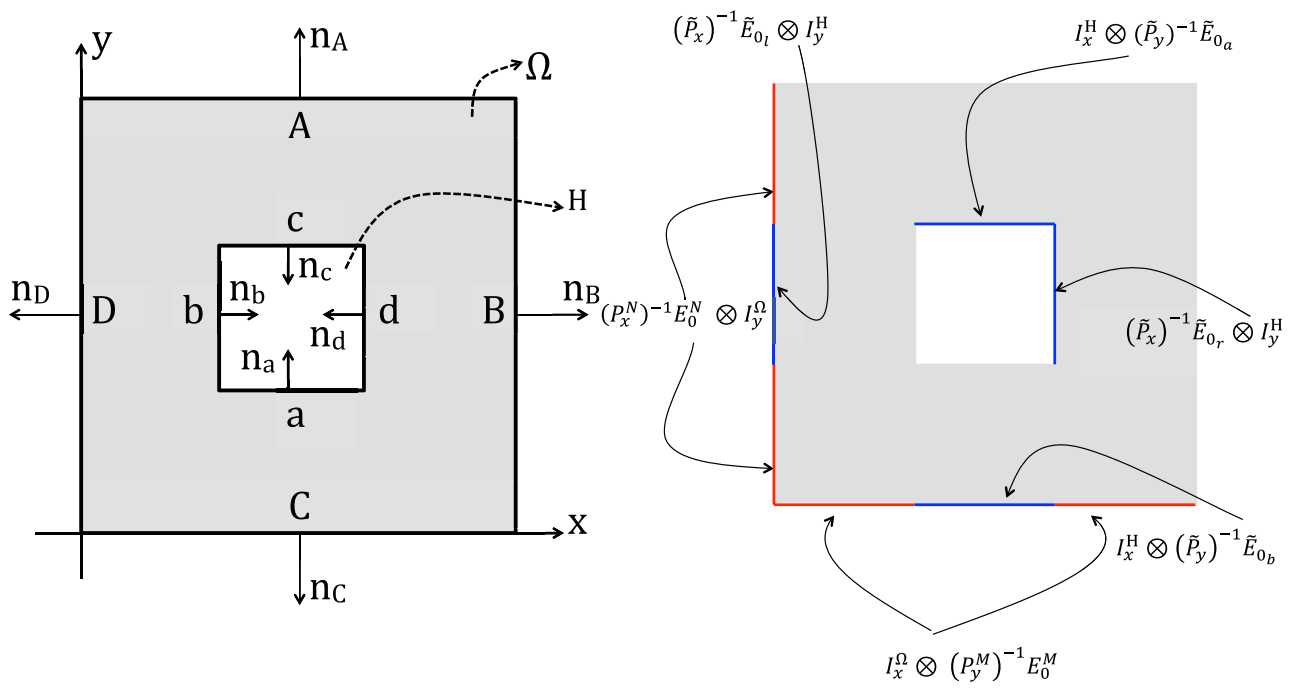

FIG. 1. (a) The model geometry, $\Omega$, its boundaries denoted by $A, B, C, D, a, b, c, d$, and the corresponding outward pointing normal vectors from each boundary. (b) A schematic of the different parts of $\delta \Omega$ influenced by the nonzero terms used in the construction of $P_{C, C, D, D}^{-1}$ in (17).

with a hole. In section 3, the discrete problem and a new combination of SBP operators are presented. Stability of the new scheme is investigated in section 4 . We extend the new approach to more complex geometries in sections 5 and 6 . Numerical calculations are shown in section 7 , where we measure the accuracy and efficiency of our scheme and compare it to the standard SBP-SAT multiblock technique. Finally, we summarize and draw conclusions in section 8.

2. Well-posedness. To develop the theory, we consider the constant coefficient scalar advection equation in two space dimensions,

$$
u_{t}+\alpha u_{x}+\beta u_{y}=0,(x, y) \in \Omega, t \in[0, T],
$$

where the subscripts $t, x$, and $y$ denote partial derivatives. The computational domain $\Omega$ is depicted in Figure 1(a). The spatial region $H$ is not a part of the computational domain; it forms a hole in $\Omega$.

The energy method (multiplying (1) with the solution and integrating over $\Omega$ ) together with the use of the Gauss-Green theorem gives

$$
\|u\|_{t}^{2}=-\oint_{\delta \Omega} u^{2}(\alpha, \beta) \cdot n d s,
$$

where $\delta \Omega=\{A \cup B \cup C \cup D \cup a \cup b \cup c \cup d\}$ is the boundary of $\Omega$. Moreover, $n=\left(n_{1}, n_{2}\right)$ is the outward pointing normal vector from $\Omega$ and $d s$ is an infinitesimal element along $\delta \Omega$. The norm is defined as $\|u\|^{2}=\iint_{\Omega} u^{2} d x d y$.

In order to bound the energy rate of the solution in (2), we specify

$$
u=g \text { if }(\alpha, \beta) \cdot n<0,
$$

where $(\alpha, \beta) \cdot n=n_{1} \alpha+n_{2} \beta$. Assuming, for example, that $\alpha, \beta>0$, (3) leads to

$$
u_{s}=g_{s} \text { and } u_{w}=g_{w},
$$


where $s \in\{C, c\}$ and $w \in\{D, d\}$; see Figure 1(a). We insert (4) into (2), integrate in time and consider an initial condition $u=f$. The continuous energy estimate becomes

$$
\|u(T)\|^{2}=\|f\|^{2}+\beta \sum_{s \in\{C, c\}} \int_{0}^{T} \int_{s} g_{s}^{2} d x d t+\alpha \sum_{w \in\{D, d\}} \int_{0}^{T} \int_{w} g_{w}^{2} d y d t+B T .
$$

In (5), $\sum$ denotes summation and $B T$ is the negative contribution from the outflow boundaries $\{A, B, a, b\}$ as

$$
B T=-\beta \sum_{n \in\{A, a\}} \int_{0}^{T} \int_{n} u^{2} d x d t-\alpha \sum_{e \in\{B, b\}} \int_{0}^{T} \int_{e} u^{2} d y d t .
$$

We summarize the result in the following.

Proposition 1. The continuous problem (1) for $\alpha, \beta>0$ augmented with boundary conditions (4) is strongly well-posed and has the bound (5).

For more details on well-posed and strongly well-posed problems, see [21].

3. Summation-by-part operators. The domain $\Phi=\{\Omega \cup H\}$ is a rectangle and we discretize it using $N$ and $M$ grid points in the $x$ and $y$ directions, respectively. In time we use $L$ time levels. The boundaries of $H$ coincide with the coordinate lines after the discretization of $\Phi$. We allocate a column vector of size $L M N$ to the grid as

$$
\mathbf{v}=\left[\begin{array}{c}
\mathbf{v}_{1} \\
\vdots \\
{\left[\mathbf{v}_{k}\right]} \\
\vdots \\
\mathbf{v}_{L}
\end{array}\right], \quad\left[\mathbf{v}_{k}\right]=\left[\begin{array}{c}
\mathbf{v}_{1} \\
\vdots \\
{\left[\mathbf{v}_{i}\right]} \\
\vdots \\
\mathbf{v}_{N}
\end{array}\right]_{k},\left[\mathbf{v}_{i}\right]_{k}=\left[\begin{array}{c}
\mathbf{v}_{1} \\
\vdots \\
\mathbf{v}_{j} \\
\vdots \\
\mathbf{v}_{M}
\end{array}\right]_{k i}
$$

in which

$$
\left\{\begin{array}{lll}
\mathbf{v}_{k i j} \approx u\left(t_{k}, x_{i}, y_{j}\right) & \text { for } \quad\left(x_{i}, y_{j}\right) \in \Omega \\
\mathbf{v}_{k i j}: \text { not defined } & \text { for } \quad\left(x_{i}, y_{j}\right) \in H .
\end{array}\right.
$$

Inside the hole $\mathbf{v}_{k i j}$ has neither any relation to the solution $u$ nor any contribution to the calculations (we will show the latter below). We have related $\mathbf{v}$ to these grid points for the convenience of using tensor products in the formulations below.

The first derivative $u_{y}$ at $x_{i}$ for all $i \in\left\{1, \ldots, N_{l}\right\} \cup\left\{N-N_{r}+1, \ldots, N\right\}$ (see Figure 2) is approximated by $D_{y}^{M} \mathbf{u}$. In Figure 2, and also in the remainder of this article, $N_{l}$ and $N_{r}$ are the number of grid points, in the $x$ direction, on the leftand right-hand sides of the hole, respectively. Additionally, $M_{a}$ and $M_{b}$ denote the number of grid points, in the $y$ direction, above and below the hole, respectively. $D_{y}^{M}$ is a so-called SBP operator of the form

$$
D_{y}^{M}=\left(P_{y}^{M}\right)^{-1} Q_{y}^{M},
$$

and $\mathbf{u}=\left[u_{1}, \ldots, u_{M}\right]^{T}$ is a smooth function injected in each grid point in the $y$ direction. The superscript $M$ denotes the size of the matrix and the subscript $y$ denotes the direction along which the operator is acting. Moreover, $P_{y}^{M}$ is a symmetric positive definite matrix, and $Q_{y}^{M}$ is an almost skew-symmetric matrix that satisfies

$$
Q_{y}^{M}+\left(Q_{y}^{M}\right)^{T}=E_{1}^{M}-E_{0}^{M}=B^{M}=\operatorname{diag}(-1,0, \ldots, 0,1) .
$$


In (10), $E_{0}^{M}=\operatorname{diag}(1,0, \ldots, 0)$ and $E_{1}^{M}=\operatorname{diag}(0, \ldots, 0,1)$. The first derivative in the $x$ direction, $D_{x}^{N}=\left(P_{x}^{N}\right)^{-1} Q_{x}^{N}$, at $y_{j}$ for all $j \in\left\{1, \ldots, M_{b}\right\} \cup\left\{M-M_{a}+1, \ldots, M\right\}$ (see Figure 2), and the first derivative in time, $D_{t}^{L}=\left(P_{t}^{L}\right)^{-1} Q_{t}^{L}$, are defined in analogous ways.

The first derivative $u_{y}$ at $x_{i}$ for all $i \in\left\{N_{l}+1, \ldots, N-N_{r}\right\}$ is approximated by $\tilde{D}_{y} \mathbf{u}$, where $\tilde{D}_{y}$ is

$$
\tilde{D}_{y}=\left[\begin{array}{ccc}
D_{y}^{M_{b}} & & \\
& 0^{M-\left(M_{a}+M_{b}\right)} & \\
& & D_{y}^{M_{a}}
\end{array}\right]
$$

In (11), $D_{y}^{M_{b}}=\left(P_{y}^{M_{b}}\right)^{-1} Q_{y}^{M_{b}}$ and $D_{y}^{M_{a}}=\left(P_{y}^{M_{a}}\right)^{-1} Q_{y}^{M_{a}}$ are the same type of SBP operators as in (9) but smaller in size ( $M_{b}$ and $M_{a}$, respectively). The notation 0 denotes a zero matrix and the superscript denotes its size (this notation is used throughout the rest of the paper). The zero matrix corresponds to the grid points inside the hole. The derivative in the $x$ direction at $y_{j}$ for all $j \in\left\{M_{b}+1, \ldots, M-M_{a}\right\}$ is constructed in the same way, as

$$
\tilde{D}_{x}=\left[\begin{array}{ccc}
D_{x}^{N_{l}} & & \\
& 0^{N-\left(N_{l}+N_{r}\right)} & \\
& & D_{x}^{N_{r}}
\end{array}\right] .
$$

A finite difference approximation including the time discretization [22, 23], on SBP form, is constructed by extending the one-dimensional SBP operators in a tensor product fashion as

$$
\begin{aligned}
& D_{t}=D_{t}^{L} \otimes\left(\left[I_{x}^{\Omega} \otimes I_{y}^{\Omega}\right]+\left[I_{x}^{H} \otimes I_{y}^{\Omega}\right]+\left[I_{x}^{\Omega} \otimes I_{y}^{H}\right]\right), \\
& D_{x}=I_{t} \otimes\left(\left[D_{x}^{N} \otimes I_{y}^{\Omega}\right]+\left[\tilde{D}_{x} \otimes I_{y}^{H}\right]\right), \\
& D_{y}=I_{t} \otimes\left(\left[I_{x}^{\Omega} \otimes D_{y}^{M}\right]+\left[I_{x}^{H} \otimes \tilde{D}_{y}\right]\right),
\end{aligned}
$$

where $I_{t}$ is the identity matrix in time and has the size $L$. In (13), $\otimes$ represents the Kronecker product which is defined as

$$
A \otimes B=\left[\begin{array}{cccc}
a_{11} B & a_{12} B & \ldots & a_{1 n} B \\
a_{21} B & a_{22} B & \ldots & a_{2 n} B \\
\vdots & \vdots & & \vdots \\
a_{m 1} B & a_{m 2} B & \ldots & a_{m n} B
\end{array}\right]
$$

for arbitrary matrices $A$ and $B$. For an $m \times n$ matrix $A$ and a $k \times l$ matrix $B$, the size of $A \otimes B$ is $(m k) \times(n l)$. More details on Kronecker products and their properties can be found in $[24,25]$. by

Moreover, $I_{y}^{H}$ and $I_{x}^{H}$ are diagonal matrices of size $M$ and $N$ respectively given

$$
I_{y}^{H}=\left[\begin{array}{lll}
0^{M_{b}} & & \\
& I^{M-\left(M_{a}+M_{b}\right)} & \\
& & 0^{M_{a}}
\end{array}\right] \text { and } I_{x}^{H}=\left[\begin{array}{lll}
0^{N_{l}} & & \\
& I^{N-\left(N_{l}+N_{r}\right)} & \\
& & 0^{N_{r}}
\end{array}\right],
$$

where $I$ (with a slight abuse of notation) denotes the identity matrix and the superscripts the size of the matrices. (In other words, $I_{x}^{H}$ and $I_{y}^{H}$ have diagonal elements 


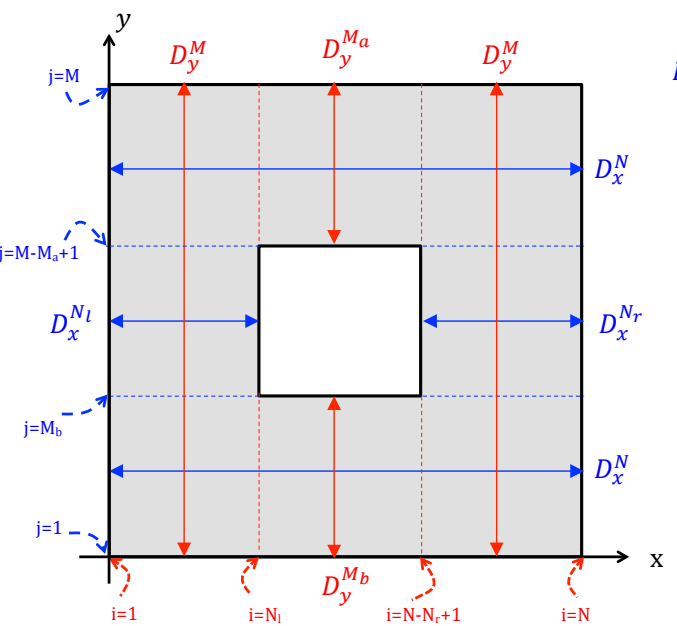

FIG. 2. A schematic of the regions where difference operators in the $x$ and $y$ directions are defined.

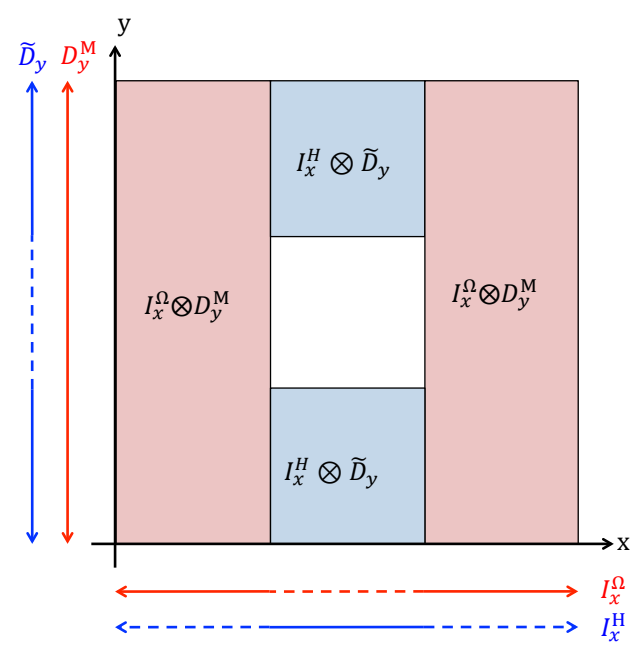

FIG. 4. A schematic that shows where the matrices, $D_{x}^{N}, \tilde{D_{x}}, I_{y}^{\Omega}$, and $I_{y}^{H}$, are defined; the solid lines correspond to the nonzero contributions.

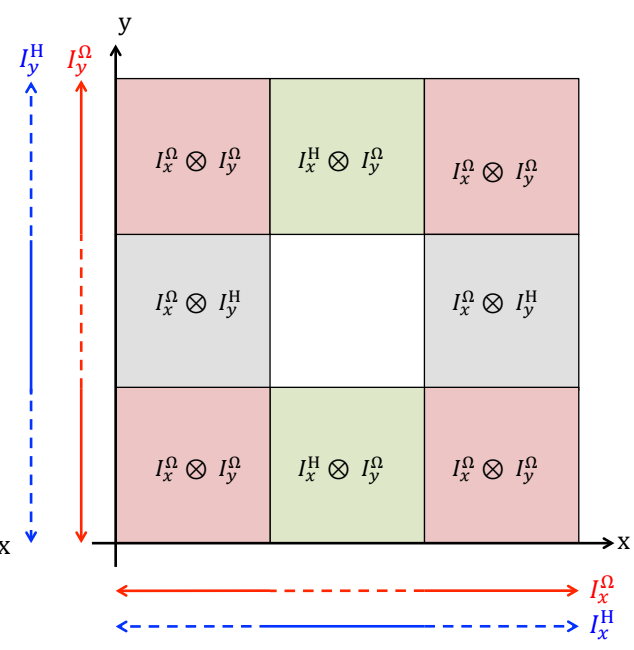

FIG. 3. A schematic of the geometry influenced with different combinations of $I_{x, y}^{\Omega}$ and $I_{x, y}^{H}$.

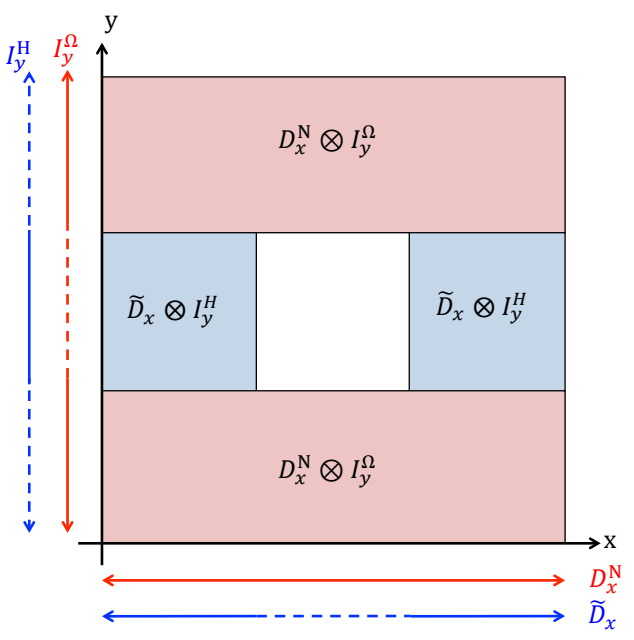

FIG. 5. A schematic that shows where the matrices, $D_{y}^{M}, \tilde{D_{y}}, I_{x}^{\Omega}$, and $I_{x}^{H}$, are defined; the solid lines correspond to the nonzero contributions.

equal to one corresponding to the points inside the hole, respectively, and zero diagonal elements otherwise.) Moreover, $I_{x}^{\Omega}=I^{N}-I_{x}^{H}$ and $I_{y}^{\Omega}=I^{M}-I_{y}^{H}$, where $I^{M, N}$ are identity matrices of size $M$ and $N$, respectively.

A schematic of different regions of $\Omega$ influenced by the different identity matrices $I_{x, y}^{\Omega}$ and $I_{x, y}^{H}$ is shown in Figure 3. Additionally, schematics of the different regions where the matrices $D_{x}$ and $D_{y}$ are active, as well as the underlying matrices involved in their construction, are shown in Figures 4 and 5.

Lemma 2. The spatial difference operators in (13) commute.

To see the proof, see [3]. 
4. Stability. The fully discrete SBP-SAT approximation of (1), including (4), can be written as

(16) $D_{t} \mathbf{v}+\alpha D_{x} \mathbf{v}+\beta D_{y} \mathbf{v}=P_{i}^{-1} \sigma_{i}\left(\mathbf{v}_{i}-\mathbf{f}\right)+\sum_{s=\{C, c\}} P_{s}^{-1} \sigma_{s}\left(\mathbf{v}_{s}-\mathbf{g}_{s}\right)+\sum_{w=\{D, d\}} P_{w}^{-1} \sigma_{w}\left(\mathbf{v}_{w}-\mathbf{g}_{w}\right)$,

where $\sigma_{i, s, w}$ are penalty coefficients for the weak initial and boundary conditions. $\mathbf{g}_{s, w}$ are zero vectors of the same size as $\mathbf{v}$, except at the positions corresponding to the inflow boundaries where the zeros are replaced with the boundary data. Moreover $\mathbf{f}$ is a zero vector, of the same size as $\mathbf{v}$, except at the positions corresponding to $t=0$ where the initial data (compatible with the boundary conditions) is injected. The subscripts $i, s$, and $w$ on the solution restrict the solution to the initial time, and the $s$ and $w$ boundary locations. Additionally,

$$
\begin{aligned}
& P_{i}^{-1}=\left(P_{t}^{L}\right)^{-1} E_{0}^{L} \otimes\left(\left[I_{x}^{\Omega} \otimes I_{y}^{\Omega}\right]+\left[I_{x}^{H} \otimes I_{y}^{\Omega}\right]+\left[I_{x}^{\Omega} \otimes I_{y}^{H}\right]\right), \\
& P_{C}^{-1}=I_{t} \otimes\left(\left[I_{x}^{\Omega} \otimes\left(P_{y}^{M}\right)^{-1} E_{0}^{M}\right]+\left[I_{x}^{H} \otimes\left(\tilde{P}_{y}\right)^{-1} \tilde{E}_{0_{b}}\right]\right), \\
& P_{c}^{-1}=I_{t} \otimes I_{x}^{H} \otimes(\tilde{P})_{y}^{-1} \tilde{E}_{0_{a}} \\
& P_{D}^{-1}=I_{t} \otimes\left(\left[\left(P_{x}^{N}\right)^{-1} E_{0}^{N} \otimes I_{y}^{\Omega}\right]+\left[\left(\tilde{P}_{x}\right)^{-1} \tilde{E}_{0_{l}} \otimes I_{y}^{H}\right]\right), \\
& P_{d}^{-1}=I_{t} \otimes\left(\tilde{P}_{x}\right)^{-1} \tilde{E}_{0_{r}} \otimes I_{y}^{H}
\end{aligned}
$$

where

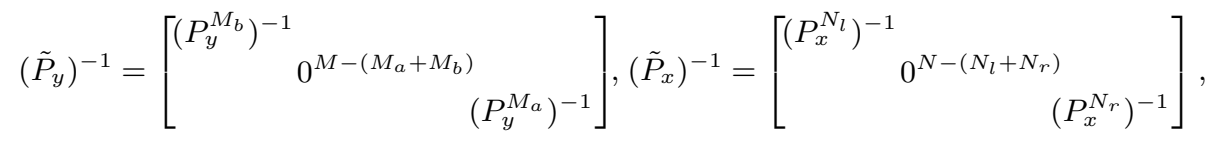

$$
\begin{aligned}
& \tilde{E}_{0_{b}}=\left[\begin{array}{ccc}
E_{0}^{M_{b}} & & \\
& 0^{M-\left(M_{a}+M_{b}\right)} & \\
& & 0^{M_{a}}
\end{array}\right], \quad \tilde{E}_{0_{a}}=\left[\begin{array}{lll}
0^{M_{b}} & & \\
& 0^{M-\left(M_{a}+M_{b}\right)} & \\
& & E_{0}^{M_{a}}
\end{array}\right], \\
& \tilde{E}_{0_{r}}=\left[\begin{array}{lll}
0^{M_{b}} & & \\
& 0^{M-\left(M_{a}+M_{b}\right)} & \\
& & E_{0}^{N_{r}}
\end{array}\right], \quad \tilde{E}_{0_{l}}=\left[\begin{array}{lll}
E_{0}^{N_{l}} & & \\
& 0^{N-\left(N_{l}+N_{r}\right)} & \\
& & 0^{N_{r}}
\end{array}\right] .
\end{aligned}
$$

Note that we have again slightly abused notation in (18) by applying the inverse sign on $\tilde{P}_{y}$ and $\tilde{P}_{x}$. In Figure $1(\mathrm{~b})$, we graphically show the different parts of $\delta \Omega$ (the inflow parts) that are influenced by nonzero contributions from the penalty terms in (17).

Next, we apply the discrete energy method, by multiplying (16) from the left with $\mathbf{v}^{T} P$, where

$$
P=P_{t}^{L} \otimes[\underbrace{\left(\left[P_{x}^{N} \otimes I_{y}^{\Omega}\right]+\left[\tilde{P}_{x} \otimes I_{y}^{H}\right]\right)}_{:=P_{x}} \underbrace{\left(\left[I_{x}^{\Omega} \otimes P_{y}^{M}\right]+\left[I_{x}^{H} \otimes \tilde{P}_{y}\right]\right)}_{:=P_{y}}],
$$

$$
\tilde{P}_{y}=\left[\begin{array}{ccc}
P_{y}^{M_{b}} & & \\
& 0^{M-\left(M_{a}+M_{b}\right)} & \\
& & P_{y}^{M_{a}}
\end{array}\right] \text { and } \tilde{P}_{x}=\left[\begin{array}{ccc}
P_{x}^{N_{l}} & & \\
& 0^{N-\left(N_{l}+N_{r}\right)} & \\
& & P_{x}^{N_{r}}
\end{array}\right] .
$$

Schematics of the different regions in the geometry influenced by $P_{x}$ and $P_{y}$, as well as the underlying matrices involved in their construction, are shown in Figures 6 and 7. By using the properties of the Kronecker product [24, 25] one can rewrite (19) as 


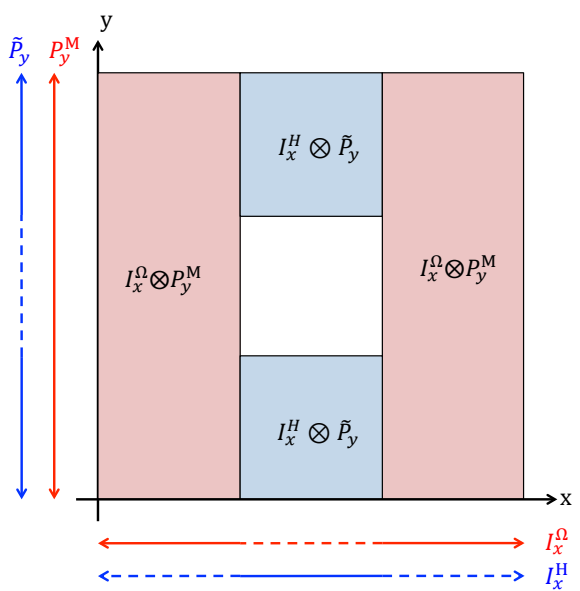

FIG. 6. A schematic that shows where the matrices, $P_{y}^{M}, \tilde{P}_{y}, I_{x}^{\Omega}$, and $I_{x}^{H}$, are defined; the solid lines correspond to the nonzero contributions.

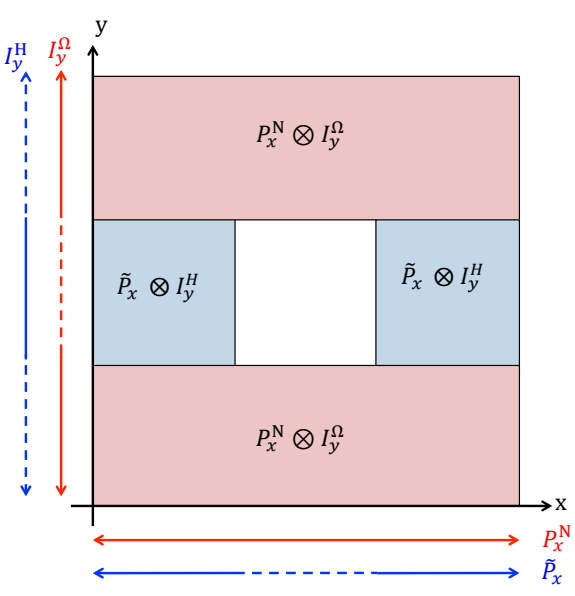

FIG. 7. A schematic that shows where the matrices, $P_{x}^{N}, \tilde{P}_{x}, I_{y}^{\Omega}$, and $I_{y}^{H}$, are defined; the solid lines correspond to the nonzero contributions.

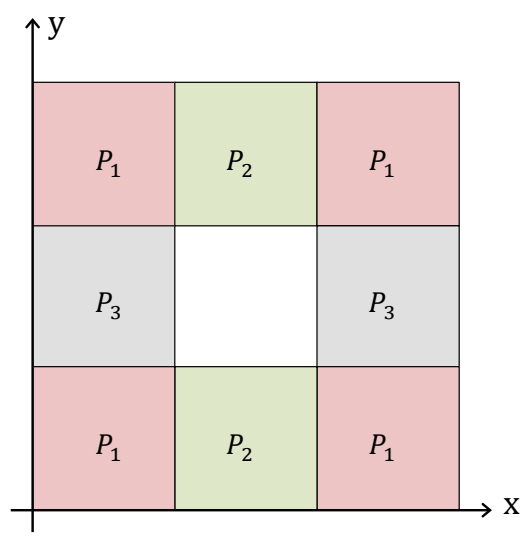

FIG. 8. A schematic of the geometry and its relation to different parts of the matrix $P$.

$$
P=P_{t}^{L} \otimes(\underbrace{I_{x}^{\Omega} P_{x}^{N} \otimes I_{y}^{\Omega} P_{y}^{M}}_{:=P_{1}}+\underbrace{I_{x}^{H} P_{x}^{N} \otimes I_{y}^{\Omega} \tilde{P}_{y}}_{:=P_{2}}+\underbrace{I_{x}^{\Omega} \tilde{P}_{x} \otimes I_{y}^{H} P_{y}^{M}}_{:=P_{3}}) .
$$

In Figure 8, the nonzero contributions from $P_{1,2,3}$ to the regions of $\Omega$ are shown.

We need the following lemma.

Lemma 3. The matrix $P$ in (21) is nonsingular.

Proof. Consider the matrix

$$
S=\left(P_{t}^{L}\right)^{-1} \otimes\left(S_{1}+S_{2}+S_{3}\right)
$$


in which

$$
\begin{aligned}
& S_{1}=I_{x}^{\Omega}\left(P_{x}^{N}\right)^{-1} \otimes I_{y}^{\Omega}\left(P_{y}^{M}\right)^{-1}, \\
& S_{2}=I_{x}^{H}\left(P_{x}^{N}\right)^{-1} \otimes I_{y}^{\Omega}\left(\tilde{P}_{y}\right)^{-1}, \\
& S_{3}=I_{x}^{\Omega}\left(\tilde{P}_{x}\right)^{-1} \otimes I_{y}^{H}\left(P_{y}^{M}\right)^{-1} .
\end{aligned}
$$

The properties of the Kronecker product give

$$
S P=I_{t} \otimes\left[\left(S_{1}+S_{2}+S_{3}\right)\left(P_{1}+P_{2}+P_{3}\right)\right] .
$$

Now, we substitute $S_{1,2,3}$ from (23) and $P_{1,2,3}$ from (21) in (24) and find

$$
\begin{aligned}
\left(S_{1}+S_{2}+S_{3}\right)\left(P_{1}+P_{2}+P_{3}\right) & =S_{1} P_{1}+S_{2} P_{2}+S_{3} P_{3} \\
& =\left(\left[I_{x}^{\Omega} \otimes I_{y}^{\Omega}\right]+\left[I_{x}^{H} \otimes I_{y}^{\Omega}\right]+\left[I_{x}^{\Omega} \otimes I_{y}^{H}\right]\right):=I^{\Omega}
\end{aligned}
$$

by the fact that $S_{i} P_{j}=0$ if $i \neq j$. In the same way, we find that $P S=I_{t} \otimes I^{\Omega}$.

Applying the discrete energy method to (16) and considering zero data gives

$$
\begin{aligned}
\mathbf{v}^{T} P D_{t} \mathbf{v}+\alpha \mathbf{v}^{T} P D_{x} \mathbf{v}+\beta \mathbf{v}^{T} P D_{y} \mathbf{v}= & \mathbf{v}^{T} P P_{i}^{-1} \sigma_{i} \mathbf{v}_{i}+\mathbf{v}^{T} P P_{C}^{-1} \sigma_{C} \mathbf{v}_{C}+\mathbf{v}^{T} P P_{c}^{-1} \sigma_{c} \mathbf{v}_{c} \\
& +\mathbf{v}^{T} P P_{D}^{-1} \sigma_{D} \mathbf{v}_{D}+\mathbf{v}^{T} P P_{d}^{-1} \sigma_{d} \mathbf{v}_{d} .
\end{aligned}
$$

Next, we evaluate the matrix products in (25) as follows:

$$
\begin{aligned}
& P D_{t}=Q_{t}^{L} \otimes\left(P_{1}+P_{2}+P_{3}\right), \\
& P D_{x}=P_{t}^{L} \otimes\left(\left[I_{x}^{\Omega} Q_{x}^{N} \otimes I_{y}^{\Omega} P_{y}^{M}\right]+\left[I_{x}^{H} Q_{x}^{N} \otimes I_{y}^{\Omega} \tilde{P}_{y}\right]+\left[I_{x}^{\Omega} \tilde{Q}_{x} \otimes I_{y}^{H} P_{y}^{M}\right]\right), \\
& P D_{y}=P_{t}^{L} \otimes\left(\left[I_{x}^{\Omega} P_{x}^{N} \otimes I_{y}^{\Omega} Q_{y}^{M}\right]+\left[I_{x}^{\Omega} \tilde{P}_{x} \otimes I_{y}^{H} Q_{y}^{M}\right]+\left[I_{x}^{H} P_{x}^{N} \otimes I_{y}^{\Omega} \tilde{Q}_{y}\right]\right),
\end{aligned}
$$

where

$$
\tilde{Q}_{x}=\left[\begin{array}{ccc}
Q_{x}^{N_{l}} & & \\
& 0^{N-\left(N_{l}+N_{r}\right)} & \\
& & Q_{x}^{N_{r}}
\end{array}\right] \text { and } \tilde{Q}_{y}=\left[\begin{array}{lll}
Q_{y}^{M_{b}} & & \\
& 0^{M-\left(M_{a}+M_{b}\right)} & \\
& & Q_{y}^{M_{a}}
\end{array}\right]
$$

Further,

$$
\begin{aligned}
& P P_{i}^{-1}=E_{0}^{L} \otimes\left(P_{1}+P_{2}+P_{3}\right):=H_{i}, \\
& P P_{C}^{-1}=P_{t}^{L} \otimes\left(\left[I_{x}^{\Omega} P_{x}^{N} \otimes I_{y}^{\Omega} E_{0}^{M}\right]+\left[I_{x}^{H} P_{x}^{N} \otimes I_{y}^{\Omega} \tilde{E}_{0_{b}}\right]\right):=H_{C}, \\
& P P_{c}^{-1}=P_{t}^{L} \otimes I_{x}^{H} P_{x}^{N} \otimes I_{y}^{\Omega} \tilde{E}_{0_{a}}:=H_{c}, \\
& P P_{D}^{-1}=P_{t}^{L} \otimes\left(\left[I_{x}^{\Omega} E_{0}^{N} \otimes I_{y}^{\Omega} P_{y}^{M}\right]+\left[I_{x}^{\Omega} \tilde{E}_{0_{l}} \otimes I_{y}^{H} P_{y}^{M}\right]\right):=H_{D}, \\
& P P_{d}^{-1}=P_{t}^{L} \otimes I_{x}^{\Omega} \tilde{E}_{0_{r}} \otimes I_{y}^{H} P_{y}^{M}:=H_{d} .
\end{aligned}
$$

The details of the computations in (26) and (28) are given in Appendix A.

By substituting (26) and (28) into (25) and adding the transpose, we obtain

$$
\begin{aligned}
\mathbf{v}^{T} H_{f} \mathbf{v}-\mathbf{v}^{T}\left(1+2 \sigma_{i}\right) H_{i} \mathbf{v}= & \left(\beta+2 \sigma_{c}\right) \mathbf{v}^{T} H_{c} \mathbf{v}+\left(\beta+2 \sigma_{C}\right) \mathbf{v}^{T} H_{C} \mathbf{v} \\
& +\left(\alpha+2 \sigma_{d}\right) \mathbf{v}^{T} H_{d} \mathbf{v}+\left(\alpha+2 \sigma_{D}\right) \mathbf{v}^{T} H_{D} \mathbf{v} \\
& +C T
\end{aligned}
$$




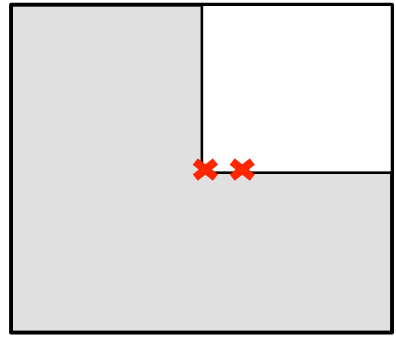

SBP21, $\mathrm{x}$ derivative

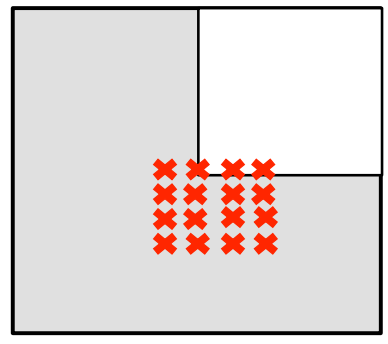

SBP42, $x$ derivative

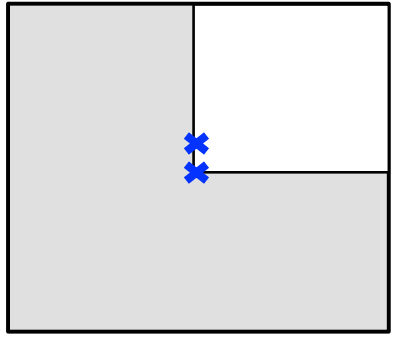

SBP21, y derivative

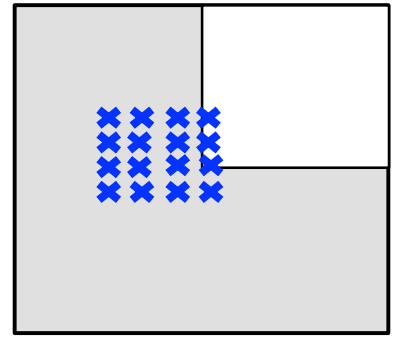

SBP42, y derivative

FIG. 9. A closer look at $C T$, near one of the corners, for SBP21 and SBP42 schemes.

TABLE 1

The size of each block in CT around one corner for schemes of different order of accuracy.

\begin{tabular}{c|c|c|c}
\hline SBP & 21 & 42 & 63 \\
\hline Size of $C T$ & $2 \times 1$ & $4 \times 4$ & $6 \times 6$ \\
\hline
\end{tabular}

where $H_{f}=E_{1}^{L} \otimes\left(P_{1}+P_{2}+P_{3}\right)$ and $C T$ stands for corner terms. Details of the derivation of (29) are given in Appendix B.

In (29), we choose $\sigma_{i}=-1, \sigma_{c, C}=-\beta$, and $\sigma_{d, D}=-\alpha$ which, in the absence of $C T$ and the presence of data, lead to

$$
\|\mathbf{v}\|_{H_{f}}^{2}=\|\mathbf{f}\|_{H_{i}}^{2}+\beta \sum_{s \in\{C, c\}}\left\|\mathbf{g}_{s}\right\|_{H_{s}}^{2}+\alpha \sum_{w \in\{D, d\}}\left\|\mathbf{g}_{w}\right\|_{H_{w}}^{2}+\mathbf{D I},
$$

where DI includes the extra damping effects from the initial and inflow boundary procedures. However, in the presence of $C T$, on the right-hand side of (30) we have an indefinite term that involves the solution on a few grid points enclosed in two small blocks around each corner. These blocks are results of using central differences in the $\xi$ and $\eta$ directions around the corners while having different norms in the perpendicular directions. Hence, the skew-symmetric property of the difference operators cannot be preserved and the interior grid contribution is not removed in $C T$. In Figure 9 we show schematically where $C T$ is located when using the SBP21 and SBP42 operators. The size of each block depends on the order of the difference operators and is independent of the number of grid points; see Table 1.

In order to investigate the stability, we consider the semidiscrete version of (1) in SBP-SAT form, written as

$$
\mathbf{v}_{t}+A \mathbf{v}_{x}=0
$$




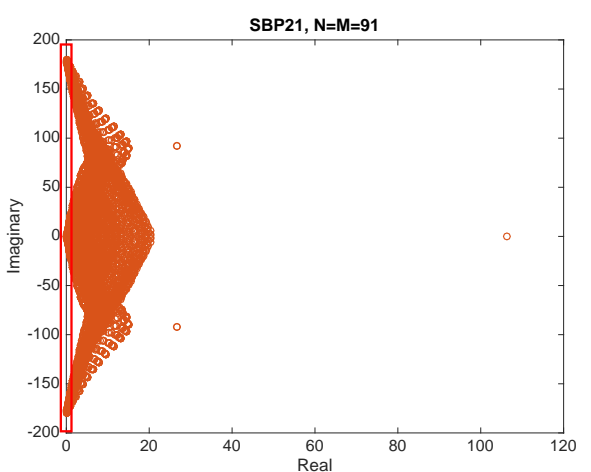

FIG. 10. The discrete spectrum for the second order case (SBP21), $N=M=91$.

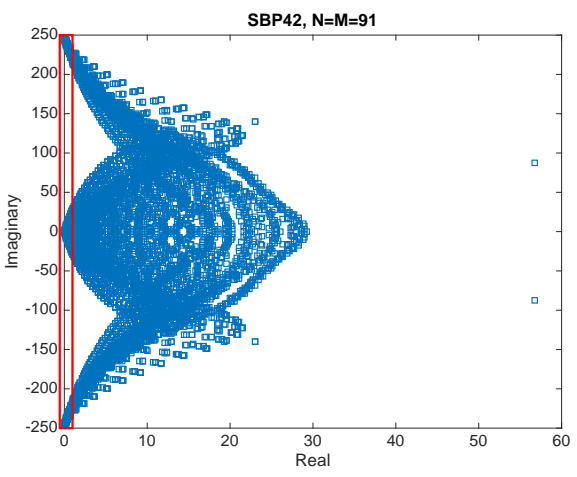

FIG. 12. The discrete spectrum for the third order case $(S B P 42), N=M=91$.

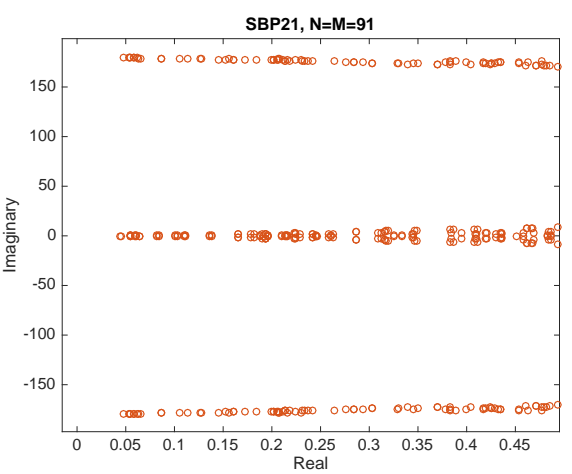

FIG. 11. A blow-up of the spectrum near the imaginary axis, $S B P 21, N=M=91$.

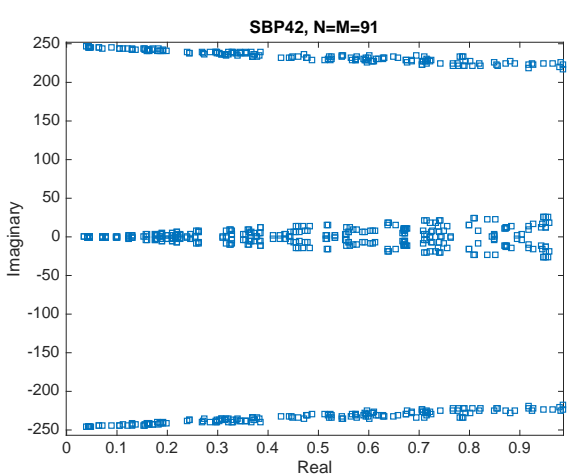

FIG. 13. A blow-up of the spectrum near the imaginary axis, $S B P 42, N=M=91$.

and investigate the spectrum of $A$. In (31), $A$ is given by

$$
A=\alpha D_{x}+\beta D_{y}-\sum_{s=\{C, c\}} P_{s}^{-1} \sigma_{s}-\sum_{w=\{D, d\}} P_{w}^{-1} \sigma_{w},
$$

and $\sigma_{s, w}$ and $P_{C, c, D, d}^{-1}$ are given in (16) (discard $I_{t}$ in (17)). We assume $\alpha=1, \beta=1$ and use the following stability conditions for the penalty parameters:

$$
1+2 \sigma_{i} \leq 0, \beta+2 \sigma_{C, c} \leq 0, \alpha+2 \sigma_{D, d} \leq 0 .
$$

We choose $\sigma_{i}=-1, \sigma_{C}=\sigma_{c}=-\beta$, and $\sigma_{D}=\sigma_{d}=-\alpha$.

The eigenvalue distribution of $A$ with SBP operators of different orders on a grid of size $91 \times 91$ are shown in Figures 10-17. The minimum real part of the spectrum for a sequence of mesh refinements is given in Figure 18. As Figures 10-18 show, the eigenvalues of $A$ for all orders of accuracy have the correct sign with a minimum real part clearly positive. In combination with SBP-SAT in time, this implies stability for these particular meshes.

5. Extension to geometries with multiple holes. One can readily extend the techniques presented in sections 3 and 4 to construct SBP operators and stable schemes for geometries with multiple holes; see Figure 19. In this example, we partition $\Omega$ along the $x$ and $y$ axes, as seen in Figure 20. The partitioning along the $x$ axis 


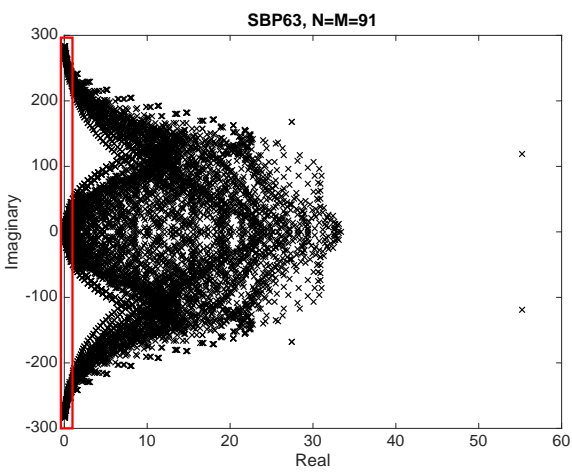

FIG. 14. The discrete spectrum for the fourth order case (SBP63), $N=M=91$.

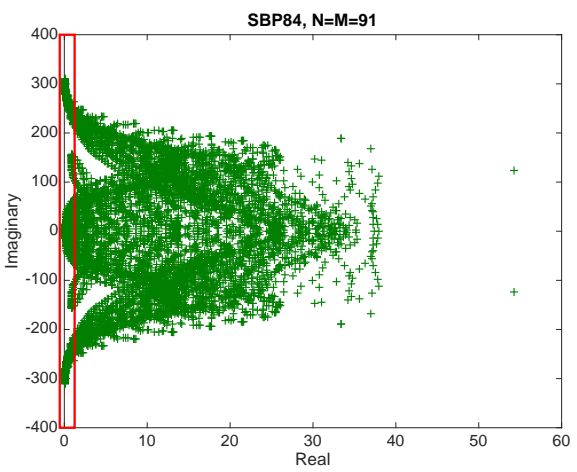

FIG. 16. The discrete spectrum for the fifth order case (SBP84), $N=M=91$.

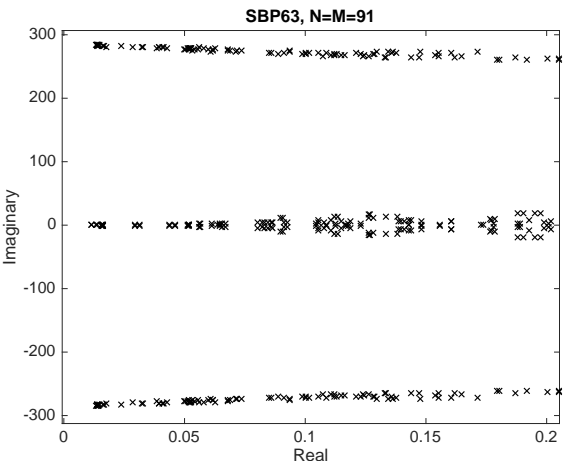

Fig. 15. A blow-up of the spectrum near the imaginary axis, SBP63, $N=M=91$.

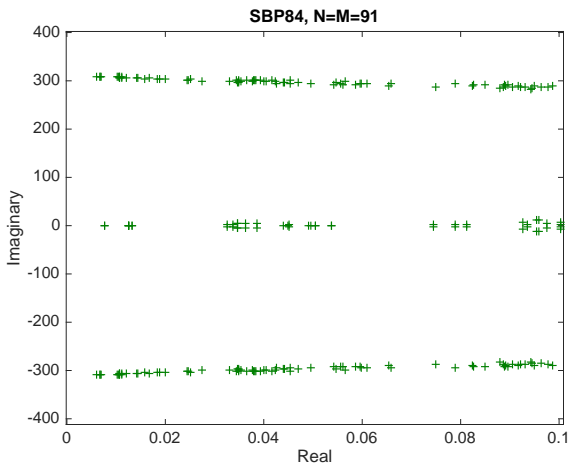

FIG. 17. A blow-up of the spectrum near the imaginary axis, $S B P 84, N=M=91$.

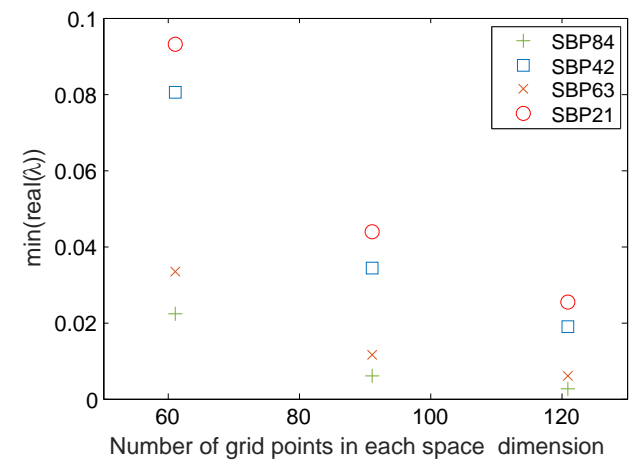

FIG. 18. The minimum real part of the spectrum for a sequence of mesh refinements for different orders of accuracy.

is done such that in each partition, only one difference operator approximates the $y$ derivative. The partitioning along the $y$ axis is analogous.

The difference operators in the $y$ and $x$ directions are defined using tensor products in the following way:

$$
D_{y}=\sum_{i=1}^{5}\left(I_{x}^{N_{i}} \otimes D_{y}^{i}\right), D_{x}=\sum_{j=1}^{5}\left(D_{x}^{j} \otimes I_{y}^{M_{j}}\right) .
$$




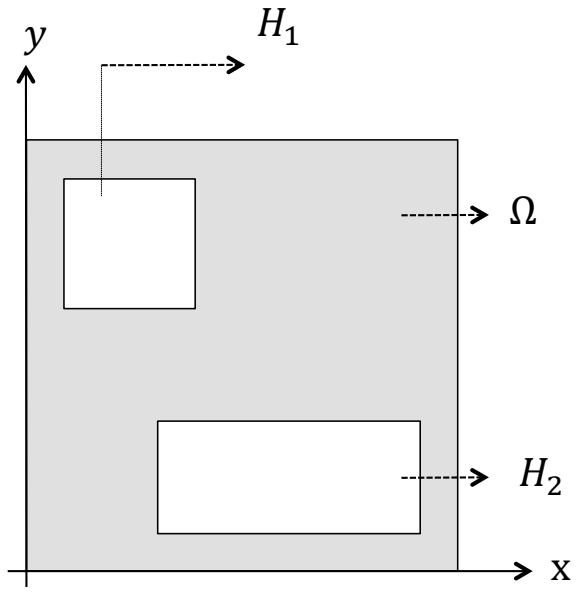

FIG. 19. A geometry with two holes.

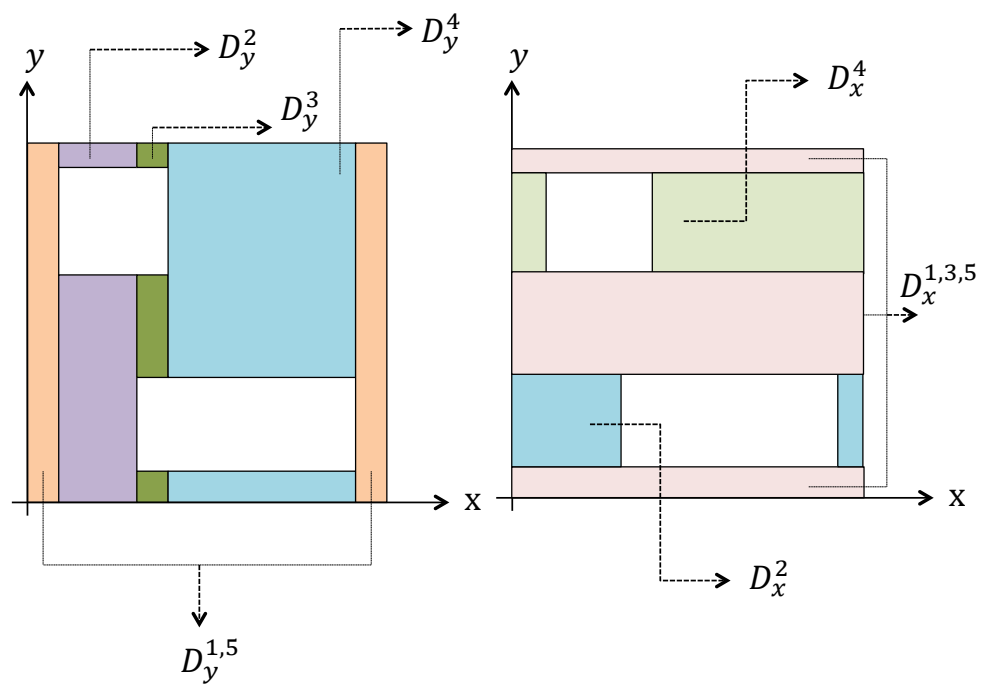

FIG. 20. A schematic of the partitioning along the $x$ and $y$ axes.

In (34), the matrices $I_{x}^{N_{i}}$ and $I_{y}^{M_{j}}$ are defined to single out the different segments on the $x$ and $y$ axes over which one partition is defined. As an example, $I_{x}^{N_{1}}$ is an $N \times N$ matrix $\left(N=\sum_{i=1}^{5} N_{i}\right)$ which has elements equal to one on the main diagonal corresponding to the $N_{1}$ grid points shown in Figure 21. Other matrices are defined similarly. Moreover, in (34), we have used

$$
\begin{aligned}
D_{y}^{1} & =\left(P_{y}^{M}\right)^{-1} Q_{y}^{M}, \\
D_{y}^{2} & =\left[\begin{array}{lll}
\left(P_{y}^{M_{1}+M_{2}+M_{3}}\right)^{-1} Q_{y}^{M_{1}+M_{2}+M_{3}} & & \\
& 0^{M_{4}} & \\
& & \left(P_{y}^{M_{5}}\right)^{-1} Q_{y}^{M_{5}}
\end{array}\right],
\end{aligned}
$$




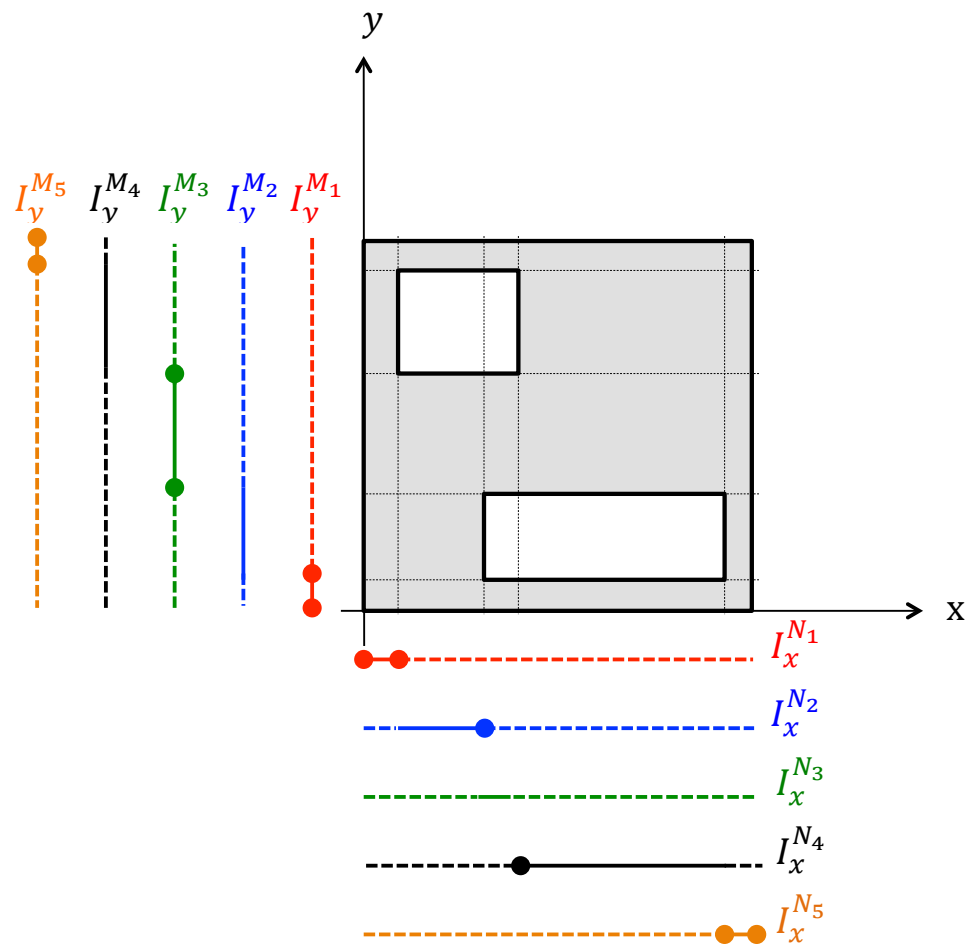

FIG. 21. A schematic of the different segments along the $x$ and $y$ axes influenced by $I_{x}^{N_{1}, N_{2}, N_{3}, N_{4}, N_{5}}$ and $I_{y}^{M_{1}, M_{2}, M_{3}, M_{4}, M_{5}}$, respectively. The superscripts denote the number of grid points in each segment; the solid lines correspond to the nonzero elements; end points are boldface if they belong to that term.

$$
\begin{aligned}
& D_{y}^{3}=\left[\begin{array}{ccccc}
\left(P_{y}^{M_{1}}\right)^{-1} Q_{y}^{M_{1}} & & & & \\
& 0^{M_{2}} & & & \\
& & \left(P_{y}^{M_{3}}\right)^{-1} Q_{y}^{M_{3}} & & \\
& & & 0^{M_{4}} & \\
& & & \left(P_{y}^{M_{5}}\right)^{-1} Q_{y}^{M_{5}}
\end{array}\right], \\
& D_{y}^{4}=\left[\begin{array}{ccc}
\left(P_{y}^{M_{1}}\right)^{-1} Q_{y}^{M_{1}} & & \\
& 0^{M_{2}} & \\
& & \left(P_{y}^{M_{3}+M_{4}+M_{5}}\right)^{-1} Q_{y}^{M_{3}+M_{4}+M_{5}}
\end{array}\right] \text {, } \\
& D_{y}^{5}=D_{y}^{1}=\left(P_{y}^{M}\right)^{-1} Q_{y}^{M} .
\end{aligned}
$$

Similarly we have

$$
\begin{aligned}
& D_{x}^{1}=\left(P_{x}^{N}\right)^{-1} Q_{x}^{N}, \\
& D_{x}^{2}=\left[\begin{array}{ccc}
\left(P_{x}^{N_{1}+N_{2}}\right)^{-1} Q_{x}^{N_{1}+N_{2}} & & \\
& 0^{N_{3}+N_{4}} & \\
& & \left(P_{x}^{N_{5}}\right)^{-1} Q_{x}^{N_{5}}
\end{array}\right] \text {, } \\
& D_{x}^{3}=D_{x}^{1}=\left(P_{x}^{N}\right)^{-1} Q_{x}^{N}, \\
& D_{x}^{4}=\left[\begin{array}{ccc}
\left(P_{x}^{N_{1}}\right)^{-1} Q_{x}^{N_{1}} & & \\
& 0^{N_{2}+N_{3}} & \\
& & \left(P_{x}^{N_{4}+N_{5}}\right)^{-1} Q_{x}^{N_{4}+N_{5}}
\end{array}\right] \text {, } \\
& D_{x}^{5}=D_{x}^{1}=\left(P_{x}^{N}\right)^{-1} Q_{x}^{N} .
\end{aligned}
$$




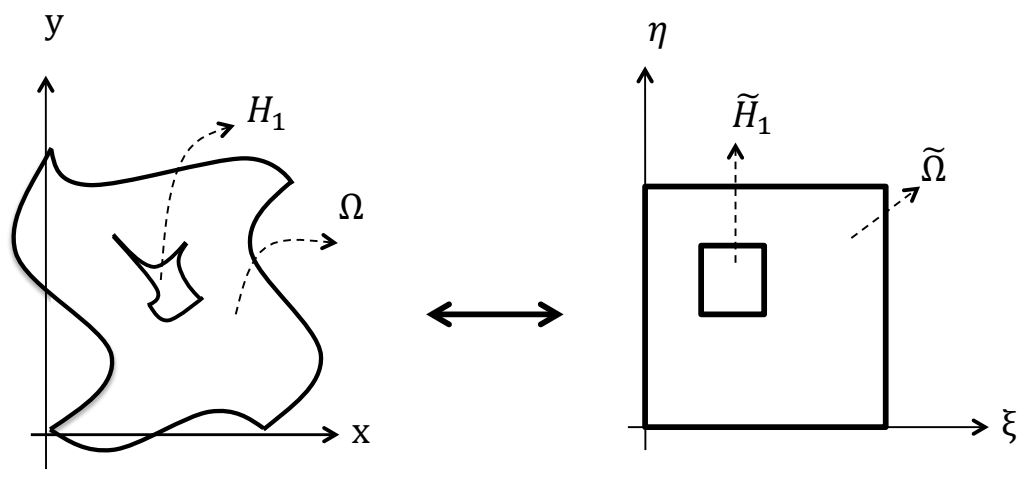

FIG. 22. A more general non-simply connected geometry in Cartesian coordinates transformed into body-fitted coordinates.

The SBP-SAT scheme is similar but more technically involved than the ones in section 3 .

6. Extension to curved geometries with holes. We extend the wellposedness analysis, SBP operators constructions, and the stability procedure presented in sections 2, 3, and 4 to a general geometry with a hole. As seen in Figure 22 , the curved computational domain is mapped into a rectangular geometry with a hole, namely, $\tilde{\Omega}$, by using a Cartesian to the body-fitted coordinate transformation $(x, y) \rightleftharpoons(\xi, \eta)$. The Jacobian matrix of the transformation is denoted by $[J]$, where

$$
[J]=\left[\begin{array}{ll}
x_{\xi} & y_{\xi} \\
x_{\eta} & y_{\eta}
\end{array}\right]
$$

and $(\partial / \partial x, \partial / \partial y)^{T}=[J](\partial / \partial \xi, \partial / \partial \eta)^{T}$. Equation (1) is then transformed to the body-fitted coordinate system, which results in a variable coefficient advection equation,

$$
J u_{t}+\tilde{\alpha}(\xi, \eta) u_{\xi}+\tilde{\beta}(\xi, \eta) u_{\eta}=0,(\xi, \eta) \in \tilde{\Omega}, t \in[0, T] .
$$

In (38), $J=x_{\xi} y_{\eta}-x_{\eta} y_{\xi}$ is the determinant of $[J], \tilde{\alpha}(\xi, \eta)=\alpha J \xi_{x}+\beta J \xi_{y}$, and $\tilde{\beta}(\xi, \eta)=\alpha J \eta_{x}+\beta J \eta_{y}$, where $\tilde{\alpha}, \tilde{\beta}$ are variables and $\alpha, \beta$ are constants.

The energy method applied to (38) together with the use of the Green-Gauss theorem in $\tilde{\Omega}$ gives

$$
\frac{d}{d t}\left(\|u\|_{J}^{2}\right)=-\oint_{\delta \tilde{\Omega}} u^{2}(\tilde{\alpha}, \tilde{\beta}) \cdot n d s,
$$

where $\delta \tilde{\Omega}=\{A \cup B \cup C \cup D \cup a \cup b \cup c \cup d\}$ is the boundary of $\tilde{\Omega}$. Moreover, $n=\left(n_{1}, n_{2}\right)$ is the outward pointing normal vector from $\tilde{\Omega}$ and $d s$ is an infinitesimal element along $\delta \tilde{\Omega}$. The norm is defined as $\|u\|_{J}^{2}=\int_{\tilde{\Omega}} J u^{2} d \xi d \eta$.

In order to bound the energy rate of the solution in (39), we specify

$$
u=g \text { if }(\tilde{\alpha}, \tilde{\beta}) \cdot n<0,
$$

where $(\tilde{\alpha}, \tilde{\beta}) \cdot n=n_{1} \tilde{\alpha}(\xi, \eta)+n_{2} \tilde{\beta}(\xi, \eta)$. For clarity and brevity, we only consider the $s$ and $w$ boundaries (see Figure $1(\mathrm{a})$ ), where $s \in\{C, c\}$ and $w \in\{D, d\}$. The boundary conditions are chosen to be 


$$
\begin{gathered}
u_{s}=g_{s} \quad \text { if } \quad \tilde{\beta}_{s}>0, \\
u_{w}=g_{w} \quad \text { if } \quad \tilde{\alpha}_{w}>0 .
\end{gathered}
$$

We insert (41) into (39), integrate in time, and consider an initial condition $u=f$. The continuous energy estimate becomes

$$
\begin{aligned}
\|u(T)\|_{J}^{2}= & \left.|| f\right|_{J} ^{2}+\frac{\tilde{\beta}_{s}+\left|\tilde{\beta}_{s}\right|}{2} \sum_{s \in\{C, c\}} \int_{0}^{T} \int_{s} g_{s}^{2} d \xi d t \\
& +\frac{\tilde{\alpha}_{w}+\left|\tilde{\alpha}_{w}\right|}{2} \sum_{w \in\{D, d\}} \int_{0}^{T} \int_{w} g_{w}^{2} d \eta d t \\
& +\frac{\tilde{\beta}_{s}-\left|\tilde{\beta}_{s}\right|}{2} \sum_{s \in\{C, c\}} \int_{0}^{T} \int_{s} u_{s}^{2} d \xi d t \\
& +\frac{\tilde{\alpha}_{w}-\left|\tilde{\alpha}_{w}\right|}{2} \sum_{w \in\{D, d\}} \int_{0}^{T} \int_{w} u_{w}^{2} d \eta d t+B T .
\end{aligned}
$$

In (5), $B T$ is the contribution from the other boundaries $\{A, B, a, b\}$ which are treated similarly to the $s$ and $w$ boundaries.

We discterize the transformed geometry using the standard technique described in section 3. However, the governing equation needs to be split $[3,4]$ prior to the discretization. The split version of (38) is

$$
J u_{t}+(\tilde{\alpha} u)_{\xi}-\tilde{\alpha}_{\xi} u+(\tilde{\beta} u)_{\eta}-\tilde{\beta}_{\eta} u=0,(\xi, \eta) \in \tilde{\Omega}, t \in[0, T] .
$$

The fully discrete SBP-SAT approximation of (43), including (41), is

$$
\begin{aligned}
& D_{t} \mathcal{J} \mathbf{v}+D_{\xi} \mathcal{A} \mathbf{v}-\mathcal{A}_{\xi} \mathbf{v}+D_{\eta} \mathcal{B} \mathbf{v}-\mathcal{B}_{\eta} \mathbf{v} \\
& =P_{i}^{-1} \sigma_{i}\left(\mathbf{v}_{i}-\mathbf{f}\right)+\sum_{s=\{C, c\}} P_{s}^{-1} \Sigma_{s}\left(\mathbf{v}_{s}-\mathbf{g}_{s}\right)+\sum_{w=\{D, d\}} P_{w}^{-1} \Sigma_{w}\left(\mathbf{v}_{w}-\mathbf{g}_{w}\right),
\end{aligned}
$$

where $\sigma_{i}$ is the initial penalty coefficient and $\Sigma_{s, w}$ are penalty matrices for the weak boundary conditions. Moreover, $\mathbf{g}_{s, w}$ are vectors of the same size as $\mathbf{v}$, with boundary data at appropriate positions and zeros elsewhere. Additionally, $\mathbf{f}$ is a zero vector, of the same size as $\mathbf{v}$, except at the positions corresponding to $t=0$ where the initial data is injected. The subscripts $i, s$, and $w$ on the solution restrict the solution to the initial time and the $s$ and $w$ boundary locations. The matrices $P_{i, s, w}^{-1}$ are defined similarly to their counterparts in (17). Finally, $\mathcal{J}, \mathcal{A}$, and $\mathcal{B}$ are diagonal matrices approximating $J, \tilde{\alpha}$, and $\tilde{\beta}$, defined by

$$
\mathcal{A}_{\xi}=D_{\xi}\left(D_{\eta} \eta \mathcal{A}-D_{\eta} \xi \mathcal{B}\right), \mathcal{B}_{\eta}=D_{\eta}\left(-D_{\xi} \eta \mathcal{A}+D_{\xi} \xi \mathcal{B}\right) .
$$

In (45), $\xi$ and $\eta$ are diagonal matrices having the $\xi$ and $\eta$ coordinates at the relevant locations on the diagonal.

The discrete energy method with zero data result in

$$
\begin{gathered}
\mathbf{v}^{T} P \mathcal{J} D_{t} \mathcal{J} \mathbf{v}+\mathbf{v}^{T} P D_{\xi} \mathcal{A} \mathbf{v}+\mathbf{v}^{T} P D_{y} \mathcal{B} \mathbf{v}+\mathbf{v}^{T} P\left(\mathcal{A}_{\xi}+\mathcal{B}_{\eta}\right) \mathbf{v}=\mathbf{v}^{T} P P_{i}^{-1} \sigma_{i} \mathbf{v}_{i} \\
+\mathbf{v}^{T} P P_{C}^{-1} \Sigma_{C} \mathbf{v}_{C}+\mathbf{v}^{T} P P_{c}^{-1} \Sigma_{c} \mathbf{v}_{c}+\mathbf{v}^{T} P P_{D}^{-1} \Sigma_{D} \mathbf{v}_{D}+\mathbf{v}^{T} P P_{d}^{-1} \Sigma_{d} \mathbf{v}_{d}
\end{gathered}
$$


Following a similar procedure as in the derivation of (26)-(28) one obtains

$$
\begin{aligned}
\mathbf{v}^{T} H_{f} \mathbf{v}+\mathbf{v}^{T} P\left(\mathcal{A}_{\xi}+\mathcal{B}_{\eta}\right) \mathbf{v}= & \mathbf{v}^{T}\left(\mathcal{B}+2 \Sigma_{c}\right) H_{c} \mathbf{v}+\mathbf{v}^{T}\left(\mathcal{B}+2 \Sigma_{C}\right) H_{C} \mathbf{v} \\
& +\mathbf{v}^{T}\left(\mathcal{A}+2 \Sigma_{d}\right) H_{d} \mathbf{v}+\mathbf{v}^{T}\left(\mathcal{A}+2 \Sigma_{D}\right) H_{D} \mathbf{v} \\
& +\mathbf{v}^{T}\left(1+2 \sigma_{i}\right) H_{i} \mathbf{v}+C T
\end{aligned}
$$

where $H_{f}=\mathcal{J}\left(E_{1}^{L} \otimes\left(P_{1}+P_{2}+P_{3}\right)\right)$ and $C T$ stands for corner terms.

As a result of Lemma $2, \mathcal{A}_{\xi}+\mathcal{B}_{\eta}=0$ (the numerical geometric conservation law holds) and hence the last term on the left-hand side of (47) vanishes.

To get an estimate as in (30), we choose $\sigma_{i}=-1, \Sigma_{c, C}=-(|\mathcal{B}|+\mathcal{B} / 2)$, and $\Sigma_{d, D}=-(|\mathcal{A}|+\mathcal{A}) / 2$. These choices together with nonzero data and disregarding $C T$ lead to

$$
\|\mathbf{v}\|_{H_{f}}^{2}=\left\|\left.\mathbf{f}\right|_{H_{i}} ^{2}+\frac{\mathcal{B}+|\mathcal{B}|}{2} \sum_{s}\right\| \mathbf{g}_{s}\left\|_{H_{s}}^{2}+\frac{\mathcal{A}+|\mathcal{A}|}{2} \sum_{w}\right\| \mathbf{g}_{w} \|_{H_{w}}^{2}+\mathbf{D I},
$$

where DI is the extra dissipation.

Remark 4. The extension to general curvilinear geometries with multiple holes of various kinds can be handled with a generalization of the technique discussed in section 5 .

7. Numerical experiments. We consider the two-dimensional constant coefficient symmetrized Euler equations [26]

$$
U_{t}+\hat{A} U_{x}+\hat{B} U_{y}=0,(x, y) \in \Omega, t \in[0, T],
$$

where $U=[\bar{c} \rho / \sqrt{\gamma} \bar{\rho}, u, v, \theta /(\bar{c} \sqrt{\gamma(\gamma-1)})]^{T}$. In (49), $\rho, u, v, \theta$, and $\gamma$ are the density, the $x$ and $y$ velocity components, the temperature, and the ratio of specific heats, respectively. An equation of state of the form $\gamma p=\bar{\rho} \theta+\rho \bar{\theta}$, where $p$ is the pressure, closes the system (49). Moreover, the bar sign denotes the state around which we have linearized. The matrices in (49) are

$$
\hat{A}=\left[\begin{array}{cccc}
\bar{u} & \bar{c} / \sqrt{\gamma} & 0 & 0 \\
\bar{c} / \sqrt{\gamma} & \bar{u} & 0 & \sqrt{\frac{\gamma-1}{\gamma}} \bar{c} \\
0 & 0 & \bar{u} & 0 \\
0 & \sqrt{\frac{\gamma-1}{\gamma}} \bar{c} & 0 & \bar{u}
\end{array}\right], \hat{B}=\left[\begin{array}{cccc}
\bar{v} & 0 & \bar{c} / \sqrt{\gamma} & 0 \\
0 & \bar{v} & 0 & 0 \\
\bar{c} / \sqrt{\gamma} & 0 & \bar{v} & \sqrt{\frac{\gamma-1}{\gamma}} \bar{c} \\
0 & 0 & \sqrt{\frac{\gamma-1}{\gamma}} \bar{c} & \bar{v}
\end{array}\right] .
$$

In the remainder of this paper, we use $\gamma=1.4, \bar{c}=2$, and $\bar{\rho}=1$.

7.1. Accuracy. To verify the order of accuracy of the scheme we use the domain in Figure $1(\mathrm{a})$, where $\Phi=[0,1] \times[0,1]$ and $H=[1 / 3,2 / 3] \times[1 / 3,2 / 3]$. We prescribe the mean velocity field to be $(\bar{u}, \bar{v})=(1,1)$ and use the manufactured solution

$$
U_{\infty}=[\sin (x-t), \cos (x-t), \sin (y-t), \cos (y-t)]^{T}
$$

for the forcing function and initial and boundary data in (49). Moreover, characteristic boundary conditions [3] are used.

We examine the scheme for SBP operators of order $2 s$ in the interior and $s$ close to the boundaries in space for $s \in\{1,2,3,4\}$. The fifth order accurate SBP operator, with $L=81$ and $T=0.1$, is used in time. The rates of convergence are shown in Figure 23. According to [27, 28, 29, 5, 30], for a scheme with first derivative SBP operators which are $2 s$ order accurate in the interior and $s$ order accurate close to the boundaries (where diagonal norms are used) should yield $s+1$ order of accuracy globally. The results in Figure 23 converge at these rates. 


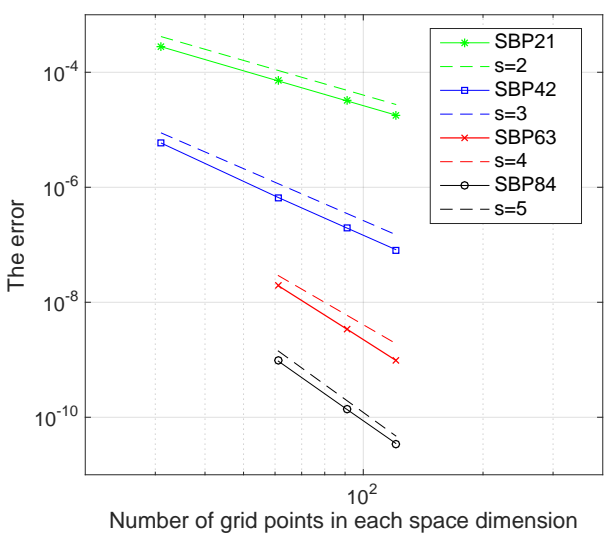

FIG. 23. Mesh refinement, the errors, and the convergence rates.

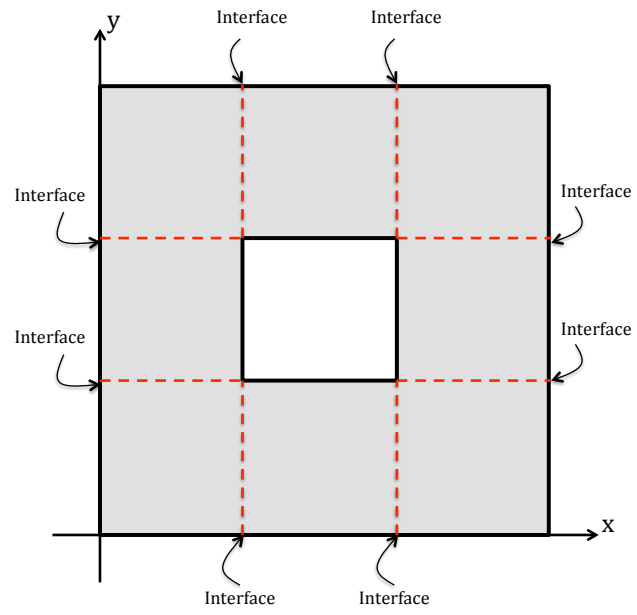

FIG. 24. A schematic of the multidomains and interfaces.

7.2. Comparison with the multiblock technique. In the standard SBPSAT multiblock approach, the domain is divided into subdomains and interface penalties are used to couple the blocks. A schematic of such multiblock division including the interfaces (dashed lines) is shown in Figure 24.

One drawback with the standard multiblock schemes is that close to interfaces, the accuracy of the approximation is reduced from $2 s$ to $s$. In order to compare this approach with our new one, we use the domain in Figure 1(a). To compare these schemes we consider the manufactured solution

$$
U_{\infty}=\left[0,0,0, e^{-10\left((x-t)^{2}+(y-t)^{2}\right)}\right]^{T} .
$$

The resulting solution is a pressure pulse that starts from $(x, y)=(0,0)$ and travels to $(x, y)=(1,1)$ as time passes.

For low order operators and coarse meshes the influence of the interfaces is visible on the solution, as seen in Figures 25-28, where the pressure distribution for different times is shown. We have used $S B P 21$ and a coarse grid of size $19 \times 19$ in space together with a fifth order SBP operator (with sufficiently small time steps) in time.

The interfaces are clearly visible in the multiblock approach, while in our approach they are not. For finer grids and higher order operators, it is more instructive to consider the error plots. For a mesh of size $61 \times 61$ and $S B P 42$ in space, together with a sufficiently accurate $S B P 84$ in time, the errors at different times are presented in Figures 29-36.

To quantitatively compare the effects of the interfaces on accuracy, the error is integrated numerically over the spatial domain. The integrated errors for the two approaches when using SBP21 and SBP42 in space are shown in Figures 37 and 39. Additionally, we show the resulting CPU time for both methods in Figures 38 and 40.

As can be seen, the gain from the new approach is twofold; we obtain lower error levels in the solution while spending less CPU time, even though the gain in CPU time for the 42 case is minimal. The reason for the large reduction in CPU time for the 21 case compared to the 42 case is presently not known to us. The convergence rates are of course identical. 


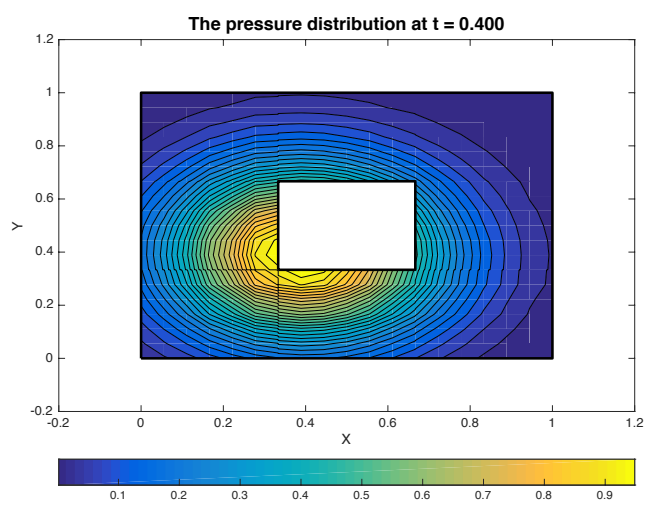

FIG. 25. The standard multiblock.

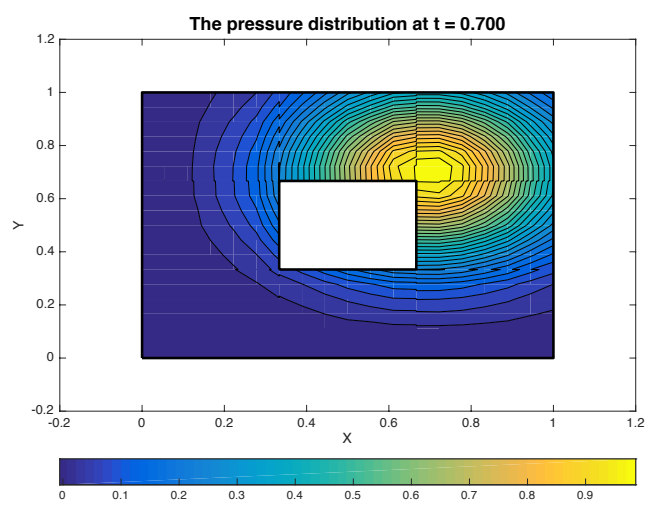

FIG. 27. The standard multiblock.

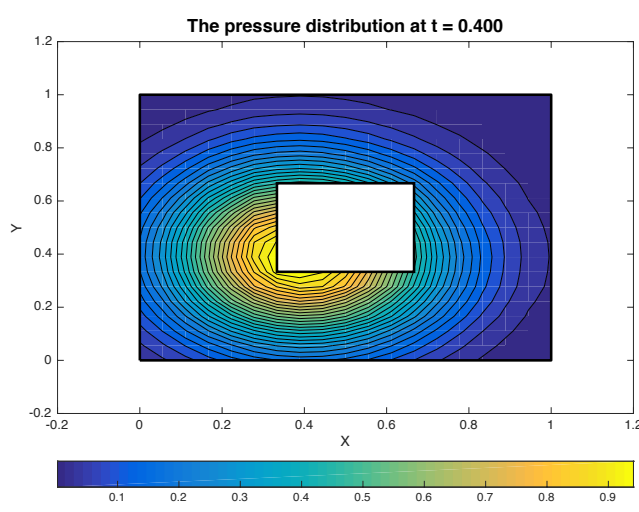

FIG. 26. The new approach.

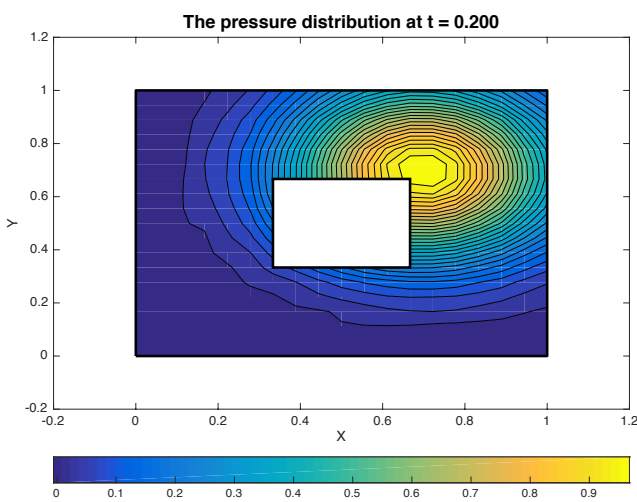

FIG. 28. The new approach.

7.3. An application. As a final application, we consider a more complex geometry where no-penetration boundary conditions are imposed on the inner solid walls. At the outer boundaries, characteristic boundary conditions [3] with data from the manufactured solution

$$
U_{\infty}=\left[0,0,0, e^{-20\left((x-t)^{2}+(y-t)^{2}\right)}\right]^{T}
$$

are imposed.

A mesh of size $51 \times 51$ grid points in space and 201 nodes in time is constructed. Third and fifth order accurate SBP operators in space and time, respectively, are used. The pressure distribution and the velocity field at different times are shown in Figures 41-48. The resulting flow is tangential to the solid inner boundaries and the pressure pulse moves out of the domain as time passes.

8. Summary and conclusions. We have constructed a new combination of summation-by-parts operators which can be applied to a number of partial differential equations posed on non-simply connected spatial domains. To develop the theory, we considered a two-dimensional constant coefficient advection equation posed on a nonsimply connected spatial domain and constructed an accurate and efficient scheme. 


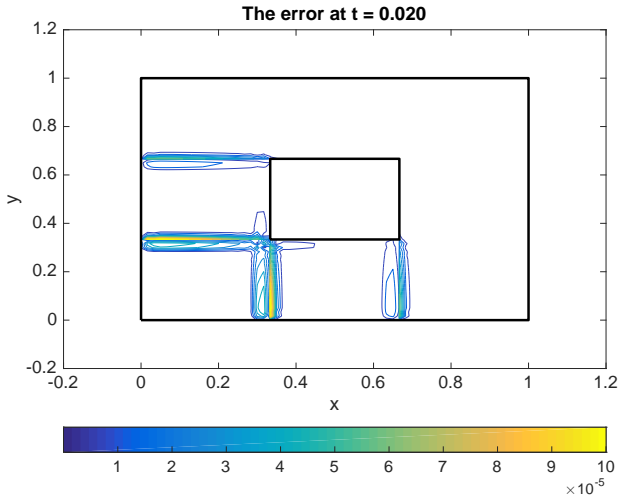

FIG. 29. The error, standard multiblock.

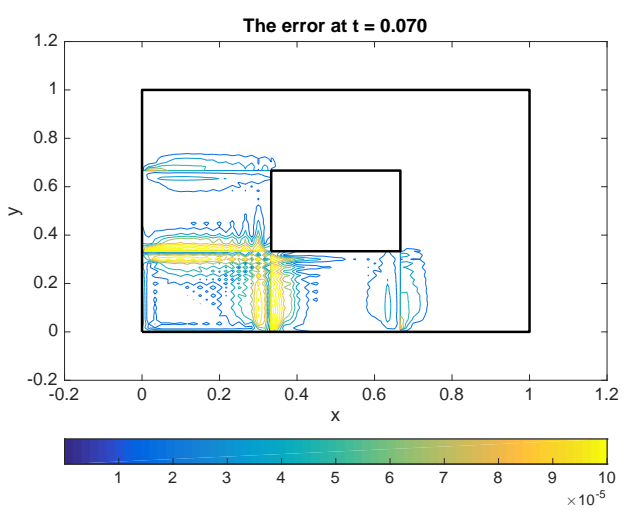

FIG. 31. The error, standard multiblock.

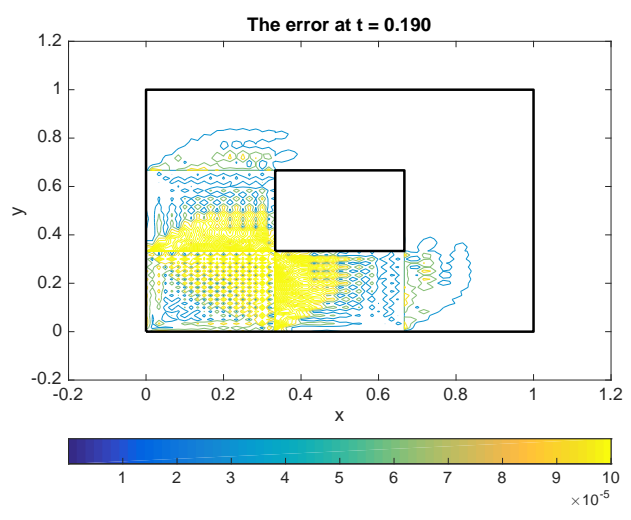

FiG. 33. The error, standard multiblock.

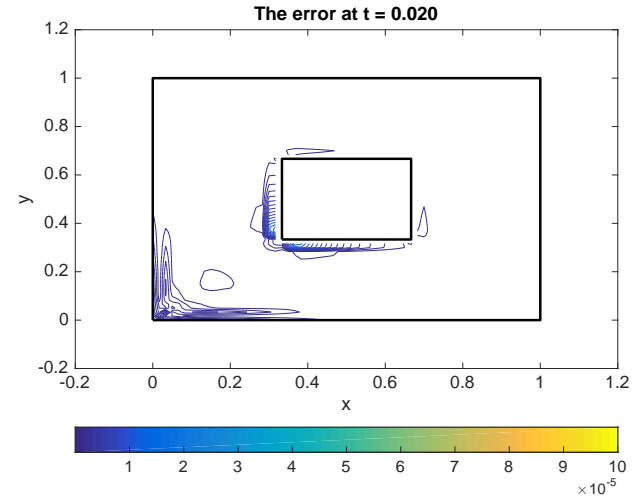

FIG. 30. The error, new approach.

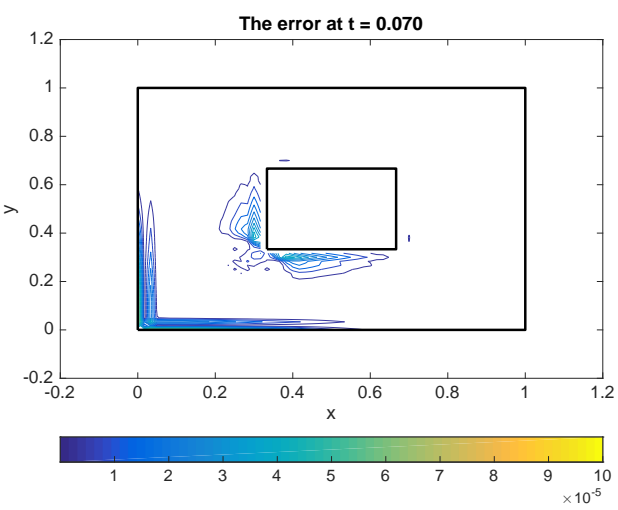

FIG. 32. The error, new approach.

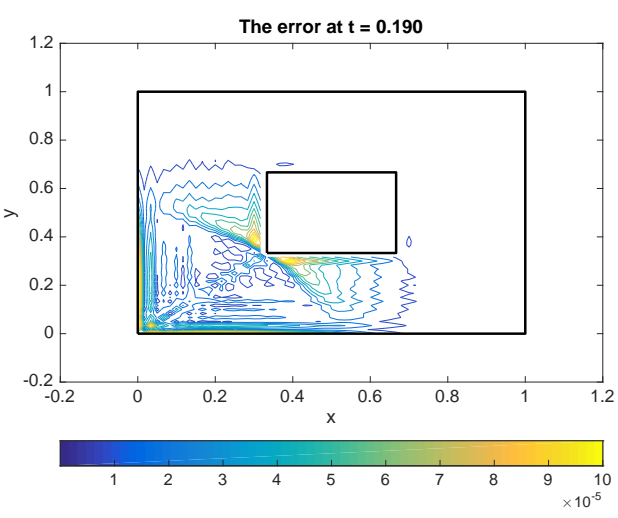

FIG. 34. The error, new approach.

Furthermore, we extended the new approach to a more complex non-simply connected geometries including curved domains. Although no proof of energy stability was obtained, correctly located spectra in combination with SBP in time indicates that the scheme is stable.

In the numerical experiments, we applied the new formulations to the linearized Euler equations. We showed that the new formulation is design order accurate by using the method of manufactured solutions. Additionally, we compared the error 


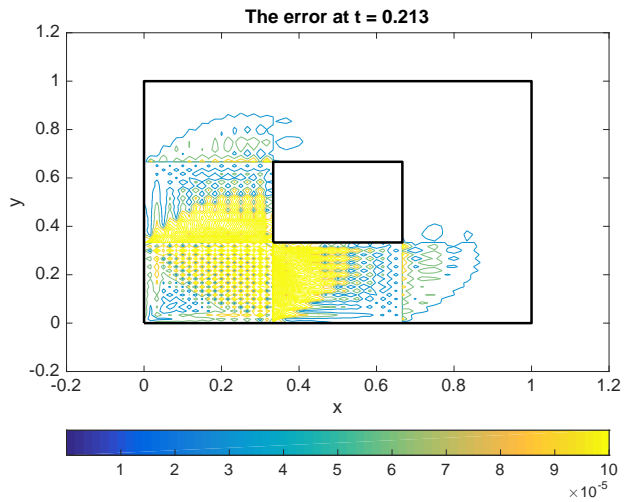

FIG. 35. The error, standard multiblock.

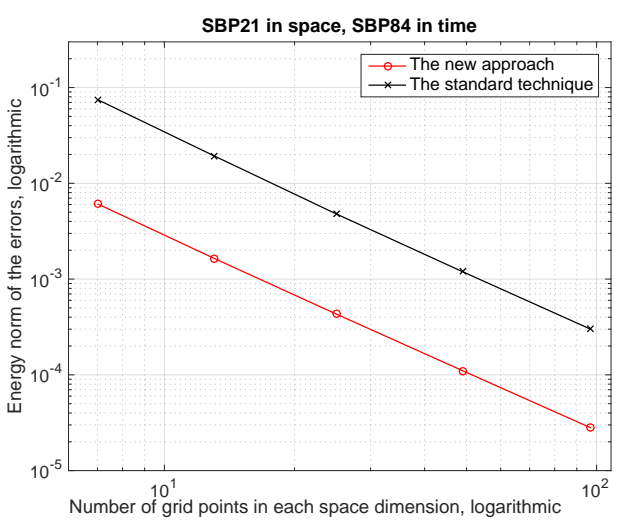

FIG. 37. Comparing the error levels.

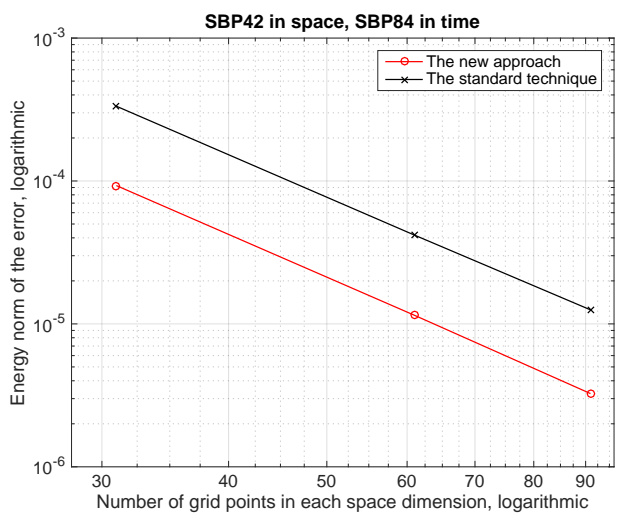

FIG. 39. Comparing the error levels.

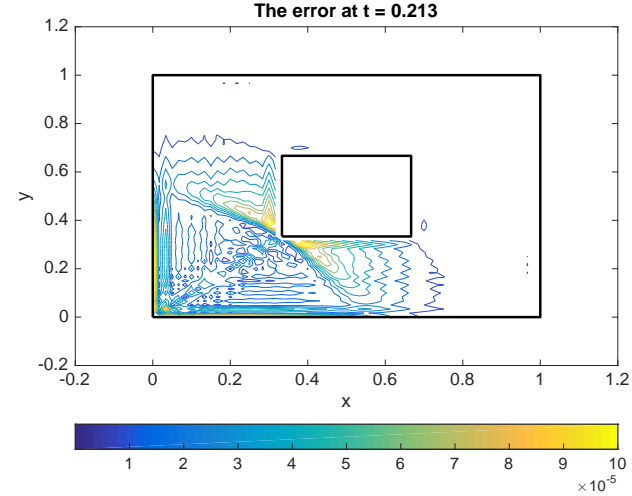

FIG. 36. The error, new approach.

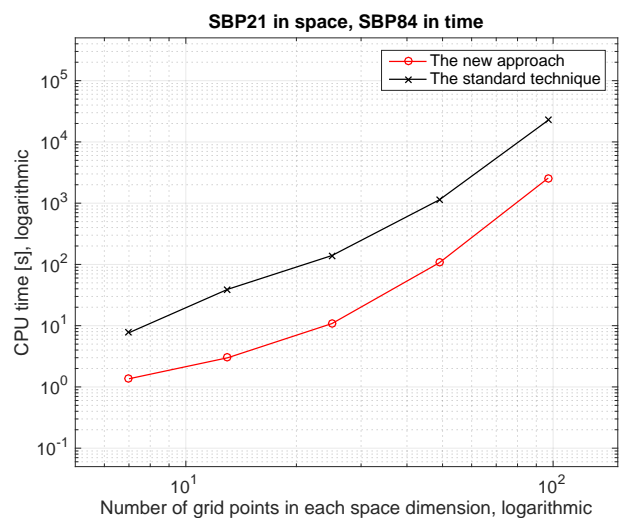

FIG. 38. Comparing the CPU times.

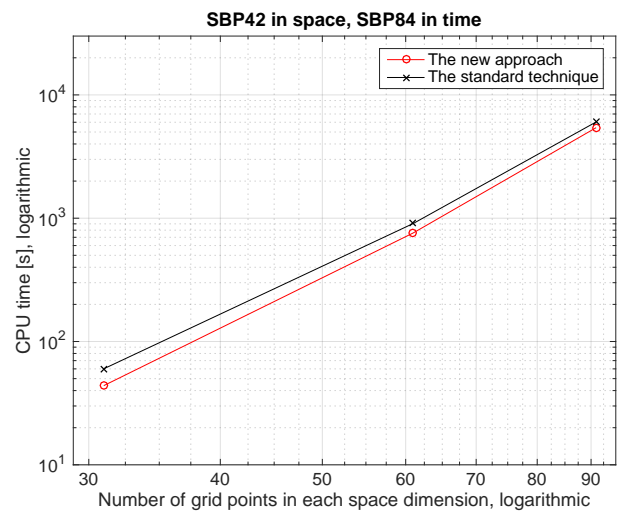

FIG. 40. Comparing the CPU times.

levels and CPU time of the new approach with the standard multiblock technique. We conclude that the new method is more accurate and efficient compared with the standard multiblock technique. An application to a more complex geometry was also presented. 


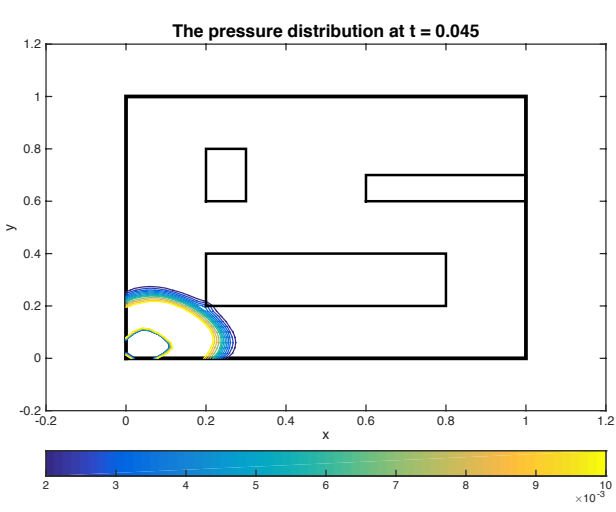

FIG. 41. The pressure distribution.

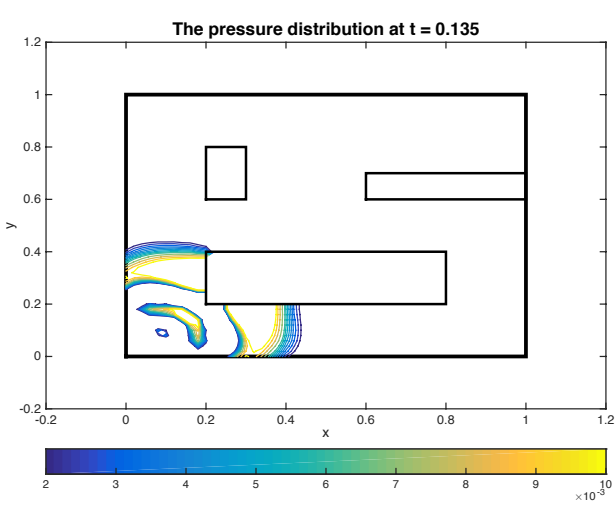

FIG. 43. The pressure distribution.

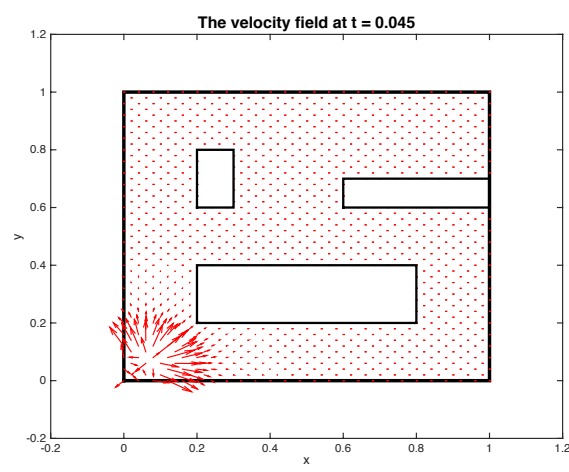

FIG. 42. The velocity field.

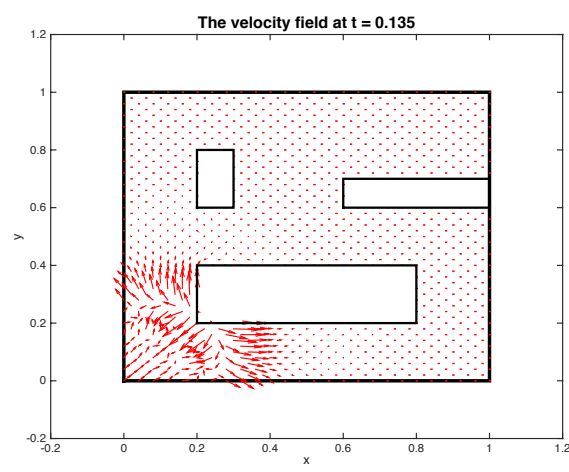

FIG. 44. The velocity field.

Appendix A. In (26) we computed the matrices as

(54)

$$
\begin{aligned}
P D_{t} & =\left[P_{t}^{L} \otimes\left(P_{1}+P_{2}+P_{3}\right)\right]\left[\left(P_{t}^{L}\right)^{-1} Q_{t}^{L} \otimes\left(\left[I_{x}^{\Omega} \otimes I_{y}^{\Omega}\right]+\left[I_{x}^{H} \otimes I_{y}^{\Omega}\right]+\left[I_{x}^{\Omega} \otimes I_{y}^{H}\right]\right)\right] \\
& =Q_{t}^{L} \otimes\left(P_{1}\left[I_{x}^{\Omega} \otimes I_{y}^{\Omega}\right]+P_{2}\left[I_{x}^{H} \otimes I_{y}^{\Omega}\right]+P_{3}\left[I_{x}^{\Omega} \otimes I_{y}^{H}\right]\right)=Q_{t}^{L} \otimes\left(P_{1}+P_{2}+P_{3}\right),
\end{aligned}
$$

$$
\begin{aligned}
P D_{x} & =\left[P_{t}^{L} \otimes\left(P_{1}+P_{2}+P_{3}\right)\right]\left[I_{t} \otimes\left(\left[\left(P_{x}^{N}\right)^{-1} Q_{x}^{N} \otimes I_{y}^{\Omega}\right]+\left[\left(\tilde{P}_{x}\right)^{-1} \tilde{Q}_{x} \otimes I_{y}^{H}\right]\right)\right] \\
& =P_{t}^{L} \otimes\left(\left(P_{1}+P_{2}\right)\left[\left(P_{x}^{N}\right)^{-1} Q_{x}^{N} \otimes I_{y}^{\Omega}\right]+P_{3}\left[\left(\tilde{P}_{x}\right)^{-1} \tilde{Q}_{x} \otimes I_{y}^{H}\right]\right) \\
& =P_{t}^{L} \otimes\left(\left[I_{x}^{\Omega} Q_{x}^{N} \otimes I_{y}^{\Omega} P_{y}^{M}\right]+\left[I_{x}^{H} Q_{x}^{N} \otimes I_{y}^{\Omega} \tilde{P}_{y}\right]+\left[I_{x}^{\Omega} \tilde{Q}_{x} \otimes I_{y}^{H} P_{y}^{M}\right]\right)
\end{aligned}
$$

and

(56)

$$
\begin{aligned}
P D_{y} & =\left[P_{t}^{L} \otimes\left(P_{1}+P_{2}+P_{3}\right)\right]\left[I_{t} \otimes\left(\left[I_{x}^{\Omega} \otimes\left(P_{y}^{M}\right)^{-1} Q_{y}^{M}\right]+\left[I_{x}^{H} \otimes\left(\tilde{P}_{y}\right)^{-1} \tilde{Q}_{y}\right]\right)\right] \\
& =P_{t}^{L} \otimes\left(\left(P_{1}+P_{3}\right)\left[I_{x}^{\Omega} \otimes\left(P_{y}^{M}\right)^{-1} Q_{y}^{M}\right]+P_{2}\left[I_{x}^{H} \otimes\left(\tilde{P}_{y}\right)^{-1} \tilde{Q}_{y}\right]\right) \\
& =P_{t}^{L} \otimes\left(\left[I_{x}^{\Omega} P_{x}^{N} \otimes I_{y}^{\Omega} Q_{y}^{M}\right]+\left[I_{x}^{\Omega} \tilde{P}_{x} \otimes I_{y}^{H} Q_{y}^{M}\right]+\left[I_{x}^{H} P_{x}^{N} \otimes I_{y}^{\Omega} \tilde{Q}_{y}\right]\right) .
\end{aligned}
$$




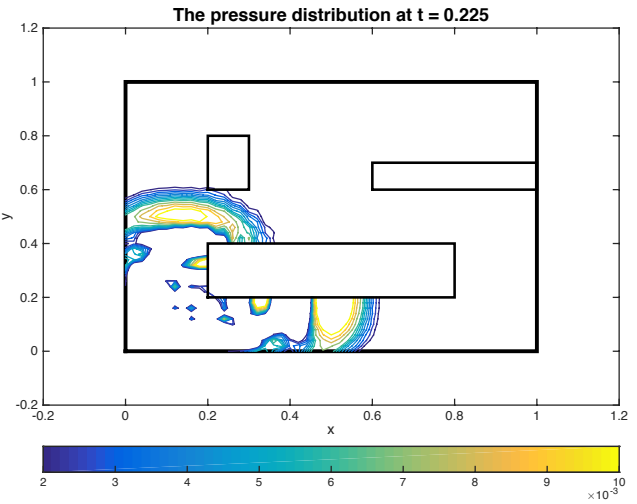

FIG. 45. The pressure distribution.

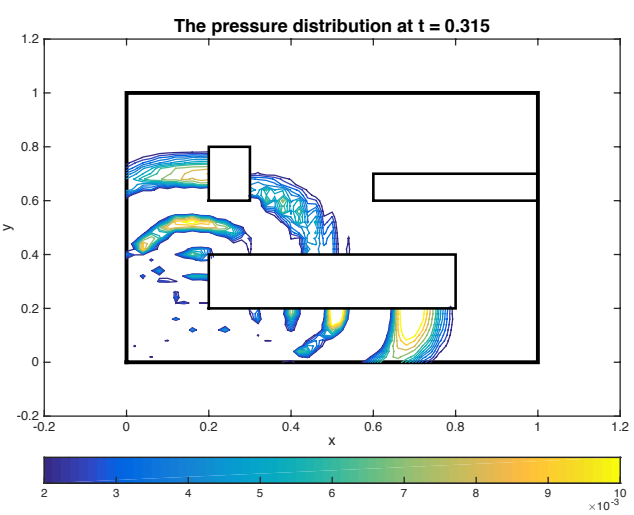

FIG. 47. The pressure distribution.

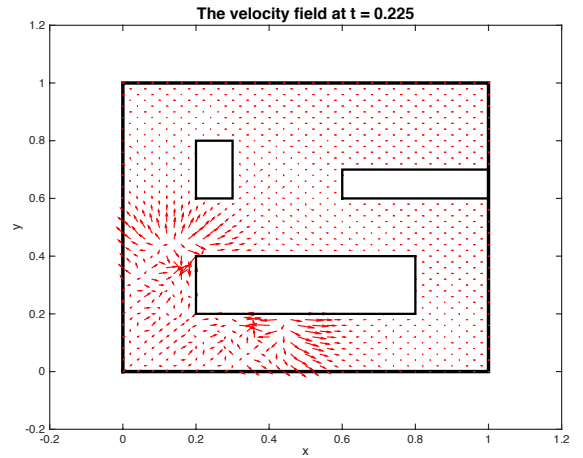

FIG. 46. The velocity field.

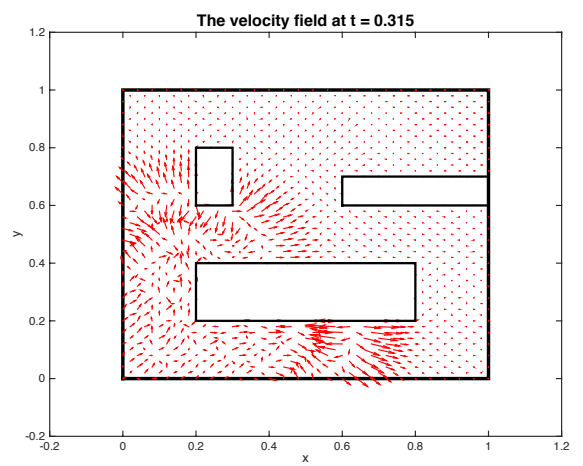

FIG. 48. The velocity field.

In (28) we used

$$
\begin{aligned}
P P_{i}^{-1} & =\left[P_{t}^{L} \otimes\left(P_{1}+P_{2}+P_{3}\right)\right]\left[\left(P_{t}^{L}\right)^{-1} E_{0}^{L} \otimes\left(\left[I_{x}^{\Omega} \otimes I_{y}^{\Omega}\right]+\left[I_{x}^{H} \otimes I_{y}^{\Omega}\right]+\left[I_{x}^{\Omega} \otimes I_{y}^{H}\right]\right)\right] \\
& =E_{0}^{L} \otimes\left(P_{1}\left[I_{x}^{\Omega} \otimes I_{y}^{\Omega}\right]+P_{2}\left[I_{x}^{H} \otimes I_{y}^{\Omega}\right]+P_{3}\left[I_{x}^{\Omega} \otimes I_{y}^{H}\right]\right)=E_{0}^{L} \otimes\left(P_{1}+P_{2}+P_{3}\right),
\end{aligned}
$$

$$
\begin{aligned}
P P_{C}^{-1} & =\left[P_{t}^{L} \otimes\left(P_{1}+P_{2}+P_{3}\right)\right]\left[I_{t} \otimes\left(\left[I_{x}^{\Omega} \otimes\left(P_{y}^{M}\right)^{-1} E_{0}^{M}\right]+\left[I_{x}^{H} \otimes\left(\tilde{P}_{y}\right)^{-1} \tilde{E}_{0_{b}}\right]\right)\right] \\
= & P_{t}^{L} \otimes\left(\left(P_{1}+P_{3}\right)\left[I_{x}^{\Omega} \otimes\left(P_{y}^{M}\right)^{-1} E_{0}^{M}\right]+P_{2}\left[I_{x}^{H} \otimes\left(\tilde{P}_{y}\right)^{-1} \tilde{E}_{0_{b}}\right]\right) \\
= & P_{t}^{L} \otimes\left(\left[I_{x}^{\Omega} P_{x}^{N} \otimes I_{y}^{\Omega} E_{0}^{M}\right]+\left[I_{x}^{H} P_{x}^{N} \otimes I_{y}^{\Omega} \tilde{E}_{0_{b}}\right]\right), \\
& \left.P P_{c}^{-1}=\left[P_{t}^{L} \otimes\left(P_{1}+P_{2}+P_{3}\right)\right]\left[I_{t} \otimes I_{x}^{H} \otimes\left(\tilde{P}_{y}\right)^{-1} \tilde{E}_{0_{a}}\right)\right] \\
& =P_{t}^{L} \otimes P_{2}\left[I_{x}^{H} \otimes\left(\tilde{P}_{y}\right)^{-1} \tilde{E}_{0_{a}}\right]=P_{t}^{L} \otimes\left[I_{x}^{H} P_{x}^{N} \otimes I_{y}^{\Omega} \tilde{E}_{0_{a}}\right],
\end{aligned}
$$

$$
P P_{D}^{-1}=\left[P_{t}^{L} \otimes\left(P_{1}+P_{2}+P_{3}\right)\right]\left[I_{t} \otimes\left(\left[\left(P_{x}^{N}\right)^{-1} E_{0}^{N} \otimes I_{y}^{\Omega}\right]+\left[\left(\tilde{P}_{x}\right)^{-1} \tilde{E}_{0_{l}} \otimes I_{y}^{H}\right]\right)\right]
$$




$$
\begin{aligned}
& =P_{t}^{L} \otimes\left(\left(P_{1}+P_{2}\right)\left[\left(P_{x}^{N}\right)^{-1} E_{0}^{N} \otimes I_{y}^{\Omega}\right]+P_{3}\left[\left(\tilde{P}_{x}\right)^{-1} \tilde{E}_{0_{l}} \otimes I_{y}^{H}\right]\right) \\
& =P_{t}^{L} \otimes\left(\left[I_{x}^{\Omega} E_{0}^{N} \otimes I_{y}^{\Omega} P_{y}^{M}\right]+\left[I_{x}^{\Omega} \tilde{E}_{0_{l}} \otimes I_{y}^{H} P_{y}^{M}\right]\right)
\end{aligned}
$$

and finally

$$
\begin{aligned}
P P_{d}^{-1} & =\left[P_{t}^{L} \otimes\left(P_{1}+P_{2}+P_{3}\right)\right]\left[I_{t} \otimes\left(\tilde{P}_{x}\right)^{-1} \tilde{E}_{0_{r}} \otimes I_{y}^{H}\right] \\
& =P_{t}^{L} \otimes P_{3}\left[\left(\tilde{P}_{x}\right)^{-1} \tilde{E}_{0_{r}} \otimes I_{y}^{H}\right]=P_{t}^{L} \otimes I_{x}^{\Omega} \tilde{E}_{0_{r}} \otimes I_{y}^{H} P_{y}^{M} .
\end{aligned}
$$

Appendix B. By substituting (26) and (28) into (25) one obtains

$$
\begin{aligned}
\mathbf{v}^{T} & {\left[Q_{t}^{L} \otimes\left(P_{1}+P_{2}+P_{3}\right)\right] \mathbf{v} } \\
& +\alpha \mathbf{v}^{T}\left[P_{t}^{L} \otimes\left(I_{x}^{\Omega} Q_{x}^{N} \otimes I_{y}^{\Omega} P_{y}^{M}+I_{x}^{H} Q_{x}^{N} \otimes I_{y}^{\Omega} \tilde{P}_{y}+I_{x}^{\Omega} \tilde{Q}_{x} \otimes I_{y}^{H} P_{y}^{M}\right)\right] \mathbf{v} \\
& +\beta \mathbf{v}^{T}\left[P_{t}^{L} \otimes\left(I_{x}^{\Omega} P_{x}^{N} \otimes I_{y}^{\Omega} Q_{y}^{M}+I_{x}^{H} P_{x}^{N} \otimes I_{y}^{\Omega} \tilde{Q}_{y}+I_{x}^{\Omega} \tilde{P}_{x} \otimes I_{y}^{H} Q_{y}^{M}\right)\right] \mathbf{v} \\
= & \sigma_{i} \mathbf{v}^{T}\left(E_{0}^{L} \otimes P^{\Omega}\right) \mathbf{v}_{i}+\sigma_{c} \mathbf{v}^{T}\left(P_{t}^{L} \otimes I_{x}^{H} P_{x}^{N} \otimes I_{y}^{\Omega} \tilde{E}_{0_{a}}\right) \mathbf{v}_{c} \\
& +\sigma_{C} \mathbf{v}^{T}\left[P_{t}^{L} \otimes\left(I_{x}^{\Omega} P_{x}^{N} \otimes I_{y}^{\Omega} E_{0}^{M}+P_{x}^{N} I_{x}^{H} \otimes I_{y}^{\Omega} \tilde{E}_{0_{b}}+\tilde{P}_{x} I_{x}^{\Omega} \otimes I_{y}^{H} E_{0}^{M}\right)\right] \mathbf{v}_{C} \\
& +\sigma_{d} \mathbf{v}^{T}\left(P_{t}^{L} \otimes I_{x}^{\Omega} \tilde{E}_{0_{r}} \otimes I_{y}^{H} P_{y}^{M}\right) \mathbf{v}_{d} \\
& +\sigma_{D} \mathbf{v}^{T}\left[P_{t}^{L} \otimes\left(I_{x}^{\Omega} E_{0}^{N} \otimes I_{y}^{\Omega} P_{y}^{M}+I_{x}^{H} E_{0}^{N} \otimes I_{y}^{\Omega} \tilde{P}_{y}+I_{x}^{\Omega} \tilde{E}_{0_{l}} \otimes I_{y}^{H} P_{y}^{M}\right)\right] \mathbf{v}_{D} .
\end{aligned}
$$

Next we add the transpose of (62) to itself. The result is

$$
\begin{aligned}
\mathbf{v}^{T} & {\left[B_{t}^{L} \otimes\left(P_{1}+P_{2}+P_{3}\right)\right] \mathbf{v} } \\
& +\alpha \mathbf{v}^{T}\left[P_{t}^{L} \otimes\left(I_{x}^{\Omega} B^{N} \otimes I_{y}^{\Omega} P_{y}^{M}+I_{x}^{H} B^{N} \otimes I_{y}^{\Omega} \tilde{P}_{y}+I_{x}^{\Omega} \tilde{B}_{x} \otimes I_{y}^{H} P_{y}^{M}\right)\right] \mathbf{v} \\
& +\beta \mathbf{v}^{T}\left[P_{t}^{L} \otimes\left(I_{x}^{\Omega} P_{x}^{N} \otimes I_{y}^{\Omega} B^{M}+I_{x}^{H} P_{x}^{N} \otimes I_{y}^{\Omega} \tilde{B}_{y}+I_{x}^{\Omega} \tilde{P}_{x} \otimes I_{y}^{H} B^{M}\right)\right] \mathbf{v} \\
= & 2 \sigma_{i} \mathbf{v}^{T}\left(E_{0}^{L} \otimes P^{\Omega}\right) \mathbf{v}_{i}+2 \sigma_{c} \mathbf{v}^{T}\left(P_{t}^{L} \otimes I_{x}^{H} P_{x}^{N} \otimes I_{y}^{\Omega} \tilde{E}_{0_{a}}\right) \mathbf{v}_{c} \\
& +2 \sigma_{C} \mathbf{v}^{T}\left[P_{t}^{L} \otimes\left(I_{x}^{\Omega} P_{x}^{N} \otimes I_{y}^{\Omega} E_{0}^{M}+P_{x}^{N} I_{x}^{H} \otimes I_{y}^{\Omega} \tilde{E}_{0_{b}}+\tilde{P}_{x} I_{x}^{\Omega} \otimes I_{y}^{H} E_{0}^{M}\right)\right] \mathbf{v}_{C} \\
& +2 \sigma_{d} \mathbf{v}^{T}\left(P_{t}^{L} \otimes I_{x}^{\Omega} \tilde{E}_{0_{r}} \otimes I_{y}^{H} P_{y}^{M}\right) \mathbf{v}_{d} \\
& +2 \sigma_{D} \mathbf{v}^{T}\left[P_{t}^{L} \otimes\left(I_{x}^{\Omega} E_{0}^{N} \otimes I_{y}^{\Omega} P_{y}^{M}+I_{x}^{H} E_{0}^{N} \otimes I_{y}^{\Omega} \tilde{P}_{y}+I_{x}^{\Omega} \tilde{E}_{0_{l}} \otimes I_{y}^{H} P_{y}^{M}\right)\right] \mathbf{v}_{D}+C T,
\end{aligned}
$$

where $\tilde{B}_{x, y}=\tilde{Q}_{x, y}+\tilde{Q}_{x, y}^{T}$. If we consider only the $s, w$ boundaries, (29) is obtained.

\section{REFERENCES}

[1] J. Nordström And M. H. CARPEnTER, High-order finite difference methods, multidimensional linear problems and curvilinear coordinates, J. Comput. Phys., 173 (2001), pp. 149-174.

[2] J. E. Kozdon, E. M. Dunham, And J. Nordström, Simulation of dynamic earthquake ruptures in complex geometries using high-order finite difference methods, J. Sci. Comput., 55 (2013), pp. 92-124.

[3] S. NikKAR AND J. NoRDSTRÖM, Fully discrete energy stable high order finite difference methods for hyperbolic problems in deforming domains, J. Comput. Phys., 291 (2015), pp. 82-98.

[4] J. Nordström, Conservative finite difference fomulations, variable coefficients, energy estimates and artificial dissipation, J. Comput. Phys., 29 (2005), pp. 345-404. 
[5] M. SVÄrd AND J. NoRdSTRÖm, Review of summation-by-parts schemes for initial-boundaryvalue problems, J. Comput. Phys., 268 (2014), pp. 17-38.

[6] J. Nordström AND J. Gong, A stable hybrid method for hyperbolic problems, J. Comput. Phys., 212 (2006), pp. 436-453.

[7] J. Gong and J. Nordström, A stable and efficient hybrid scheme for viscous problems in complex geometries, J. Comput. Phys., 226 (2007), pp. 1291-1309.

[8] J. Nordström, F. Ham, M. Shoeybi, E. Van der Weide, M. Svärd, K. Mattson, G. IACCARino, And J. Gong, A hybrid method for unsteady inviscid fluid flow, Comput. \& Fluids, 38 (2009), pp. 875-882.

[9] O. O'Reilly, J. Nordström, J. E. Kozdon, and E. M. Dunham, Simulation of earthquake rupture dynamics in complex geometries using coupled finite difference and finite volume methods, Commun. Comput. Phys., 17 (2015), pp. 337-370.

[10] D. J. Bodony, G. Zagaris, A. Reichert, and Q. Zhang, Reprint of: Aeroacoustic prediction in complex geometries, Proc. IUTAM, 1 (2010), pp. 234-243.

[11] A. Reichert, M. T. Health, And D. J. Bodony, Energy stable numerical methods for hyperbolic partial differential equations using overlapping domain decomposition, J. Comput. Phys., 1 (2012), pp. 5243-5265.

[12] W. D. Henshaw And D. W. Schwendeman, Moving overlapping grids with adaptive mesh refinement for high-speed reactive and non-reactive flow, J. Comput. Phys., 216 (2006), pp. $744-779$.

[13] N. A. Pettersson, Hole-cutting for three-dimensional overlapping grids, SIAM J. Sci. Comput., 21 (1999), pp. 646-665.

[14] J. Nordström, J. Gong, E. Van der Weide, And M. SvÄrd, A stable and conservative high order high order multi-block method for the compressible Navier-Stokes equations, J. Comput. Phys., 228, (2009), pp. 9020-9035.

[15] J. E. Kozdon and L. C. Wilcox, Provably Stable, General Purpose Projection Operators for High-Order Finite Difference Methods, arXiv:1410.5746, (2014).

[16] A. Nissen, G. Kreiss, and M. Gerritsen, Stability at nonconforming grid interfaces for a high order discretization of the Schrödinger equation, J. Sci. Comput., 53 (2012), pp. 528-551.

[17] K. Mattsson AND M. H. CARPEnter, Stable and accurate interpolation operators for high-order multi-block finite-difference methods, SIAM J. Sci. Comput., 32 (2010), pp. 2298-2320.

[18] J. Nordström, K. Forsberg, C. Adamsson, and P. Eliasson, Finite volume methods, unstructured meshes and strict stability for hyperbolic problems, Appl. Numer. Math., 45 (2003), pp. 453-473.

[19] J. E. Hicken, D. C. D. R. Fernández, And D. W. ZingG, Multidimensional Summation-byParts Operators: General Theory and Application to Simplex Elements, arXiv:1505.03125, 2015.

[20] G. J. Gassner, A skew-symmetric discontinuous Galerkin spectral element discretization and its relation to SBP-SAT finite difference methods, SIAM J. Sci. Comput., 35 (2013), A1233-A1253.

[21] B. Gustafsson, H.-O. Kreiss, and J. Oliger, Time Dependent Problems and Difference Methods, John Wiley \& Sons, New York, 1995.

[22] J. Nordström And T. Lundquist, Summation-by-parts in time, J. Comput. Phys., 251 (2013), pp. 487-499.

[23] T. Lundquist and J. Nordström, The SBP-SAT technique for initial value problems, J. Comput. Phys., 270 (2014), pp. 86-104.

[24] C. F. VAn LoAn, The ubiquitous Kronecker product, J. Comput. and Appl. Math., 123 (2000), pp. 85-100.

[25] H. Zhang And F. Ding, On the Kronecker products and their applications, J. Appl. Math., (2013), 296185.

[26] E. Turkel, Symmetrization of the fluid dynamics matrices with applications, Math. Comput., 27 (1973), pp. 729-736.

[27] B. Strand, Summation by parts for finite difference approximations of $d / d x$, J. Comput. Phys., 110 (1994), pp. 47-67.

[28] B. Gustafsson, The convergence rate for difference approximations to general mixed initialboundary value problems, SIAM J. Numer. Anal., 18 (1981), pp. 179-190.

[29] B. Gustafsson, The convergence rate for difference approximations to mixed initial boundary value problems, Math. Comp., 29 (1975), pp. 396-406.

[30] B. Gustafsson, High Order Difference Methods for Time Dependent PDE, Springer Ser. Comput. Math. 38, Springer-Verlag, New York, 2008. 